\title{
ON THE CONTROLLABILITY OF THE ROLLING PROBLEM ONTO THE HYPERBOLIC $n$-SPACE
}

\author{
YACINE CHITOUR Université Paris-Sud 11 \\ MAURICIO GODOY MOLINA Universidad de La Frontera \\ PETRI KOKKONEN
}

\begin{abstract}
In the present paper, we study the controllability of the control system associated to rolling without slipping or spinning of a Riemannian manifold $(M, g)$ onto the hyperbolic $n$-space $\mathbb{H}^{n}$. Our main result states that the system is completely controllable if and only if $(M, g)$ is not isometric to a warped product of a special form, in analogy to the classical de Rham decomposition theorem for Riemannian manifolds. The proof is based on the observations that the controllability issue in this case reduces to determine whether $(M, g)$ admits a reducible action of a hyperbolic analog of the holonomy group and a well-known fact about connected subgroups of $\mathrm{O}(n, 1)$ acting irreducibly on the Lorentzian space $\mathbb{R}^{n, 1}$.
\end{abstract}

\section{Contents}

1. Introduction

2. Notations and previous results 4

2.1. The rolling problem 4

2.2. Global properties of $\mathcal{D}_{\mathrm{R}}$-orbits 5

2.3. Space forms and their isometry groups 5

2.4. Reduction of the rolling problem 6

3. Warped products 7

4. Presentation of the main results 8

4.1. Proof of the main result 9

$\begin{array}{lr}\text { 4.2. } & \text { Proof of Proposition } 4.3 \\ 4\end{array}$

$\begin{array}{lr}\text { 4.3. Proof of Proposition 4.4 } & 19\end{array}$

$\begin{array}{ll}\text { References } & 20\end{array}$

\section{INTRODUCTION}

A major problem in geometric control theory is to give easy-to-check conditions for the controllability of the system under study. In the general geometric setting, the most common way to proceed is to verify the Lie Algebraic Rank Condition (LARC) which is a well-known sufficient condition for controllability: all the Lie brackets of the vector fields steering the dynamics have to span the tangent bundle

2000 Mathematics Subject Classification. 53C07, 53C29, 53A55, 53B30.

Key words and phrases. controllability, nonlinear geometric control, warped product, holonomy, rolling model, space forms.

The work of the first author is supported by the ANR project GCM, program "Blanche", (project number NT09_504490) and the DIGITEO-Région Ile-de-France project CONGEO. The work of the second author is partially supported by the ERC Starting Grant 2009 GeCoMethods. The work of the third author is supported by Finnish Academy of Science and Letters, KAUTE Foundation and l'Institut français de Finlande. 
of the state space. As simple as this algebraic condition may seem, in practice, however, this condition turns out to be sometimes cumbersome to verify. Kalman's controllability rank condition is a celebrated result in linear control theory that can be seen as the holy grail for this point of view: reducing a priori lengthy and difficult Lie algebraic computations to checking that a matrix has full rank. In order to find a simple criterion for controllability without computing Lie brackets, one is often forced to use strongly the geometric structure of the problem at hand, therefore, it is necessary to employ differential geometric language and tools to achieve that goal.

Our aim is to give a good geometric setting to study the controllability of the system consisting of an oriented connected Riemannian manifold of dimension $n \geq 2$ rolling without spinning or slipping against an $n$-dimensional space form of negative sectional curvature. The study of the problem of two manifolds rolling in such a way is old and provides surprisingly many difficulties. It is possible to trace back the case of the ball rolling onto the plane to Chaplygin $[8,9]$ in the end of the 19th century, though more modern accounts of the higher dimensional case for embedded manifolds has been presented by Nomizu [22] and Sharpe [25]. A coordinate-free definition for surfaces was introduced in [2, 7], and later extended to general manifolds in $[11,14]$. A general procedure to study the controllability of the rolling model has been studied in $[11,16]$.

It was observed in [12], that the structure of the affine holonomy group characterizes the orbits of the rolling problem, when one of the manifolds involved is the Euclidean space, thus one can fully address the problem of complete controllability for the rolling model. To state this observation precisely, let us recall the definition of the rolling problem. Let $(M, g)$ and $(\hat{M}, \hat{g})$ be two oriented Riemannian manifolds of dimension $n$. The configuration space of the rolling problem is the manifold

$$
\begin{aligned}
& Q=Q(M, \hat{M})=\left\{A:\left.\left.T\right|_{x} M \rightarrow T\right|_{\hat{x}} \hat{M} \mid\right. x \in M, \hat{x} \in \hat{M}, \\
&A \text { linear isometry, } \operatorname{det}(A)>0\} .
\end{aligned}
$$

An absolutely continuous curve $q(t)=(\gamma(t), \hat{\gamma}(t), A(t))$ in $Q$ is a rolling curve if $A(t) X(t)$ is parallel along $\hat{\gamma}(t)$ for every vector field $X(t)$ that is parallel along $\gamma(t)$ (no twist condition) and if $A(t) \dot{\gamma}(t)=\dot{\hat{\gamma}}(t)$ (no slip condition). There is a distribution $\mathcal{D}_{\mathrm{R}}$ on $Q$, called the rolling distribution, such that the rolling curves in $Q$ are exactly the integral curves of $\mathcal{D}_{\mathrm{R}}$. Lie brackets of vector fields spanning $\mathcal{D}_{\mathrm{R}}$ are expressed in terms of the curvature tensors $R$ and $\hat{R}$ associated to the Riemannian metrics $g$ on $M$ and $\hat{g}$ on $\hat{M}$ respectively, together with the covariant derivatives of $R$ and $\hat{R}$. It seems therefore impossible to solve for general dimension $n$ the controllability issue on the sole knowledge of the Lie algebraic structure of $\mathcal{D}_{\mathrm{R}}$, except for low dimensions (for $n=2$ this was achieved in [2,7], and for $n=3$ in [11, Section 7]). Indeed, in the case for instance where $(\hat{M}, \hat{g})$ is the $n$-dimensional Euclidean space, it would amount to determine $\operatorname{Hol}\left(\nabla^{g}\right)$, the holonomy group of the Levi-Civita connection $\nabla^{g}$ associated to $g$, with the only knowledge of its curvature tensor and its covariant derivatives. Instead, the latter issue can be successfully addressed by resorting on group theoretic and algebraic arguments.

In general, it is not clear if there is a $G$-principal bundle structure on $Q$ making $\mathcal{D}_{\mathrm{R}}$ a $G$-principal bundle connection. However, this is indeed the case for the projection $Q(M, \hat{M}) \rightarrow M$, when $(\hat{M}, \hat{g})$ is a space form, as shown in [12]. More precisely, if $(\hat{M}, \hat{g})$ has constant sectional curvature $c$, there is a Lie group $G_{c}(n)$ acting on 
$Q$ such that $\mathcal{D}_{\mathrm{R}}$ is a $G_{c}(n)$-principal bundle connection, and moreover, its fibers of orbits are all conjugate to the holonomy group of $\mathcal{D}_{\mathrm{R}}$, which is a subgroup of $G_{c}(n)$.

In the case where $c=0$, we have $G_{0}(n)=\mathrm{SE}(n)$ and this construction reduces to study the affine holonomy group of $M$. One of the main results in [12] shows that, provided $(M, g)$ is complete and $(\hat{M}, \hat{g})$ is the Euclidean space $\mathbb{R}^{n}$ with the standard Riemannian structure, then the rolling system is controllable if and only if $M$ has full holonomy, i.e., $\operatorname{Hol}\left(\nabla^{g}\right)=S O(n)$. This fact can be seen as a manifestation of De Rham's decomposition theorem since, if the holonomy of $M$ is reducible, one can detect the components of $M$ via the irreducible orbits of the distribution $\mathcal{D}_{\mathrm{R}}$.

Up to rescaling, the cases remaining are when $c= \pm 1$ and, in these cases, $G_{1}(n)=$ $\mathrm{SO}(n+1)$ and $G_{-1}(n)=\mathrm{SO}_{0}(n, 1)$, the identity component of $\mathrm{O}(n, 1)$. In both situations the controllability for the rolling system can be phrased in terms of the holonomy of a connection. As shown in [12], there is a metric $h$ and an $h$-metric connection $\nabla^{c}$ on the vector bundle $T M \oplus \mathbb{R}$ over $M$ such that the rolling system is controllable if and only if $\mathcal{H}^{c}$, the holonomy group of $\nabla^{c}$, is equal to $G_{c}(n)$. In the case $c=1$, the aforementioned metric $h$ is positive definite; whereas in the case $c=-1$, the metric $h$ has index one.

The case $c=1$ was addressed in [12]: it is shown that if the action of $\mathcal{H}^{1}$ on the unit sphere is not transitive, then $(M, g)$ is the unit sphere. As a consequence, it holds that, for $n \geq 16$ and even, the rolling system $Q=Q\left(M, S^{n-1}\right)$ is completely controllable if and only if $(M, g)$ is not isometric to the unit sphere. It is also important to stress that the results we present here do not correspond to the ones obtained in [26]. In that reference, the main result consists of an isometric decomposition of a semi-Riemannian manifold into the direct product of semi-Riemannian irreducible submanifolds.

In the present paper, we characterize the structure of a complete and simply connected Riemannian manifold $(M, g)$ in terms of the rolling system $Q=Q\left(M, \mathbb{H}^{n}\right)$. Our main result states that the action of $\mathcal{H}^{-1}$ is reducible if and only if there exists a complete simply connected Riemannian manifold $\left(M_{1}, g_{1}\right)$, so that $(M, g)$ is a warped product either of the form

(WP1): $\left(\mathbb{R} \times M_{1}, \mathrm{~d} s^{2} \oplus_{e^{-s}} g_{1}\right)$, or

(WP2): $\left(\mathbb{H}^{k} \times M_{1}, \mathbf{g}_{-1}^{k} \oplus_{\cosh (d(\cdot))} g_{1}\right)$,

where for each $x \in \mathbb{H}^{k}, d(x)$ is the distance between $x$ and an arbitrary fixed point $x_{0} \in \mathbb{H}^{k}$, and $1 \leq k \leq n$. This can be seen as a "hyperbolic" analogue of the classical de Rham decomposition theorem for Riemannian manifolds, see [19, 24]. By the classification theorem due to Berger in [5] (and also proved directly in [13]), the only connected subgroup of the Lorentz group $\mathrm{O}(n, 1)$ that acts irreducibly on the Lorentzian space $\mathbb{R}^{n, 1}$ is its identity component $\mathrm{SO}_{0}(n, 1)$. This irreducibility criterion, together with our results, implies that the rolling system $Q=Q\left(M, \mathbb{H}^{n}\right)$ is not controllable if and only if $(M, g)$ decomposes, up to isometry, into a warped product of the form (WP1) or (WP2). An interesting fact is that related results have been obtained in the study of cones over pseudo-Riemannian manifolds, see [4], with differential geometric motivations. Note also that the embedded case of two spaces of constant sectional curvature rolling on each other has been analyzed in [18].

The structure of the paper is the following. In Section 2 we collect results concerning the rolling problem that will be relevant in the proof of the main result. We give special emphasis to the extra symmetry that appears in the system when one of the manifolds is a space form. In Section 3, we recall the definition of warped products, how to detect them and how to find their warping functions through a criterion due 
to Hiepko [17]. Finally, in Section 4, we present the main results of the paper and their proofs. These results, in global formulation, consist of the decomposition of $(M, g)$ into a warped product of the form (WP1) or (WP2) (see Theorem 4.1), under the assumption that the action of $\mathcal{H}^{-1}$ is reducible. Both proofs are divided in two cases, depending whether a non-trivial subspace $V_{1}$ of $T M \oplus \mathbb{R}$, invariant under the action of $\mathcal{H}^{-1}$, contains a lightlike vector or not.

\section{Notations And PREvious Results}

Unless otherwise stated, all manifolds under consideration are smooth, connected, oriented, of finite dimension $n \geq 2$ and endowed with a Riemannian metric. Similarly, all frames will be assumed to be positively oriented.

We intend to formulate some of the results in this paper by means of the rolling formalism presented in $[11,14]$. In order to do this, we need to introduce the state space $Q=Q(M, \hat{M})$ for the rolling problem of two $n$-dimensional connected, oriented smooth Riemannian manifolds $(M, g),(\hat{M}, \hat{g})$ as

$$
\begin{aligned}
& Q=Q(M, \hat{M})=\left\{A:\left.\left.T\right|_{x} M \rightarrow T\right|_{\hat{x}} \hat{M} \mid\right. x \in M, \hat{x} \in \hat{M}, \\
&A \text { linear isometry, } \operatorname{det}(A)>0\} .
\end{aligned}
$$

The case in which $\hat{M}=\mathbb{R}^{n}$ reduces to the study of the well-known concept of antidevelopment of curves, as observed in [15]. The main idea is to lift appropriately the information about the manifold $M$ rolling onto $\mathbb{R}^{n}$ to the frame bundle $F M$. In the general case, the situation is more complicated.

2.1. The rolling problem. For $q=(x, \hat{x} ; A) \in Q$ and $\left.X \in T\right|_{x} M$ we define the rolling lift $\left.\left.\mathscr{L}_{\mathrm{R}}(X)\right|_{q} \in T\right|_{q} Q$ as

$$
\left.\mathscr{L}_{\mathrm{R}}(X)\right|_{q}=\left.\frac{\mathrm{d}}{\mathrm{d} t}\right|_{0}\left(P_{0}^{t}(\hat{\gamma}) \circ A \circ P_{t}^{0}(\gamma)\right),
$$

where $\gamma, \hat{\gamma}$ are any smooth curves in $M, \hat{M}$, respectively, such that $\dot{\gamma}(0)=X$ and $\dot{\hat{\gamma}}(0)=A X$, and $P_{a}^{b}(\gamma)$ (resp. $P_{a}^{b}(\hat{\gamma})$ ) denotes the parallel transport along $\gamma$ from $\gamma(a)$ to $\gamma(b)$ (resp. along $\hat{\gamma}$ from $\hat{\gamma}(a)$ to $\hat{\gamma}(b)$ ).

The rolling distribution $\mathcal{D}_{\mathrm{R}}$ on $Q$ is the $n$-dimensional smooth distribution defined, for $q=(x, \hat{x} ; A) \in Q$, by

$$
\left.\mathcal{D}_{\mathrm{R}}\right|_{q}=\left.\mathscr{L}_{\mathrm{R}}\left(\left.T\right|_{x} M\right)\right|_{q} .
$$

An absolutely continuous (a.c. for short) curve $t \mapsto q(t)=(\gamma(t), \hat{\gamma}(t) ; A(t))$ is a rolling curve if and only if it is a.e. tangent to the distribution $\mathcal{D}_{\mathrm{R}}$. See $[11,14]$ for a description using local coordinates. We use $\mathcal{O}_{\mathcal{D}_{\mathrm{R}}}(q)$ to denote the $\mathcal{D}_{\mathrm{R}}$-orbit passing through $q$. Also, if $q_{0}=\left(x_{0}, \hat{x}_{0} ; A_{0}\right) \in Q$ and $\gamma:[a, b] \rightarrow M$ is a curve such that $\gamma(a)=x_{0}$, we let $q_{\mathcal{D}_{\mathrm{R}}}\left(\gamma, q_{0}\right)(t), t \in\left[a, b^{\prime}\right]$, to be the unique rolling curve through $q_{0}$ that projects to $\gamma$ on $M$ Here $b^{\prime} \leq b$ in general; if $(M, g)$ is complete, then one can take $b=b^{\prime}$. See $[12,19]$.

Remark 2.1 The use of the adjective "rolling" in the previous definitions has its origin in the classical kinematic model of one Riemannian manifold rolling onto another one of the same dimension, without spinning or slipping (cf. [2, 3, 11, 14, 25]). This kinematic model can be traced back to the definition of holonomy by É. Cartan (cf. [6]) and has important applications in robotics (e.g. the plate-ball problem $[1,20,21])$. The main idea in this formulation is that each point $(x, \hat{x} ; A)$ of the state space $Q$ can be viewed as describing a contact point of the two manifolds which is given by the points $x$ and $\hat{x}$ 
of $M$ and $\hat{M}$, respectively, and an isometry $A$ of the tangent spaces $\left.T\right|_{x} M,\left.T\right|_{\hat{x}} \hat{M}$ at this contact point, measuring the relative orientation of these tangent spaces. An a.c. curve $t \mapsto q(t)=(\gamma(t), \hat{\gamma}(t) ; A(t))$ in $Q$ is a rolling curve if the following constraints (see e.g. [2], [3, Chapter 24], [10]) are satisfied

(i) The no-spinning condition: for every a.c. curve $[a, b] \rightarrow T M ; t \mapsto X(t)$ of vectors along $t \mapsto \gamma(t)$, we have

$$
\nabla_{\dot{\gamma}(t)} X(t)=0 \quad \Longrightarrow \quad \hat{\nabla}_{\dot{\hat{\gamma}}(t)}(A(t) X(t))=0 \quad \text { for a.e. } t \in[a, b] .
$$

(ii) The no-slipping condition:

$$
A(t) \dot{\gamma}(t)=\dot{\hat{\gamma}}(t) \quad \text { for a.e. } t \in[a, b] .
$$

2.2. Global properties of $\mathcal{D}_{\mathrm{R}}$-orbits. An important technical result shown in [12] is the action of Riemannian isometries of $M$ and $\hat{M}$ on the state space $Q$.

Proposition 2.2 Let $F \in \operatorname{Iso}(M, g)$ and $\hat{F} \in \operatorname{Iso}(\hat{M}, \hat{g})$ be Riemannian isometries of $(M, g)$ and $(\hat{M}, \hat{g})$ respectively. Define smooth free right and left actions of $\operatorname{Iso}(M, g)$, $\operatorname{Iso}(\hat{M}, \hat{g})$ on $Q$ by

$$
\begin{aligned}
q_{0} \cdot F & :=\left(F^{-1}\left(x_{0}\right), \hat{x}_{0} ;\left.A_{0} \circ F_{*}\right|_{F^{-1}\left(x_{0}\right)}\right), \\
\hat{F} \cdot q_{0} & :=\left(x_{0}, \hat{F}\left(\hat{x}_{0}\right) ;\left.\hat{F}_{*}\right|_{\hat{x}_{0}} \circ A_{0}\right),
\end{aligned}
$$

where $q_{0}=\left(x_{0}, \hat{x}_{0} ; A_{0}\right) \in Q$. We also set $\hat{F} \cdot q_{0} \cdot F:=\left(\hat{F} \cdot q_{0}\right) \cdot F=\hat{F} \cdot\left(q_{0} \cdot F\right)$. Then for any $q_{0}=\left(x_{0}, \hat{x}_{0} ; A_{0}\right) \in Q$, a.c. $\gamma:[0,1] \rightarrow M, \gamma(0)=x_{0}$, and $F \in \operatorname{Iso}(M, g)$, $\hat{F} \in \operatorname{Iso}(\hat{M}, \hat{g})$, one has, for all $t \in[0,1]$,

$$
\hat{F} \cdot q_{\mathcal{D}_{\mathrm{R}}}\left(\gamma, q_{0}\right)(t) \cdot F=q_{\mathcal{D}_{\mathrm{R}}}\left(F^{-1} \circ \gamma, \hat{F} \cdot q_{0} \cdot F\right)(t) .
$$

In particular, $\hat{F} \cdot \mathcal{O}_{\mathcal{D}_{\mathrm{R}}}\left(q_{0}\right) \cdot F=\mathcal{O}_{\mathcal{D}_{\mathrm{R}}}\left(\hat{F} \cdot q_{0} \cdot F\right)$.

An initial reduction of the problem is the fact that the controllability question for the rolling problem for $M$ and $\hat{M}$ is equivalent to study the controllability for the rolling problem for Riemannian coverings of $M$ and $\hat{M}$ (cf. [11]). An immediate consequence, is that one can assume with no loss of generality that both manifolds $M$ and $\hat{M}$ are simply connected.

2.3. Space forms and their isometry groups. The $n$-dimensional space form $\mathbb{F}_{c}^{n}$ of curvature $c \neq 0$ as a subset of $\mathbb{R}^{n+1}, n \geq 1$, given by

$$
\mathbb{F}_{c}^{n}:=\left\{\left(x_{1}, \ldots, x_{n+1}\right) \in \mathbb{R}^{n+1} \mid c\left(x_{1}^{2}+\cdots+x_{n}^{2}\right)+x_{n+1}^{2}=1, x_{n+1}+\frac{c}{|c|} \geq 0\right\} .
$$

Equip $\mathbb{F}_{c}^{n}$ with a Riemannian metric $\mathbf{g}_{c}^{n}$ defined as the restriction to $\mathbb{F}_{c}^{n}$ of the nondegenerate symmetric $(0,2)$-tensor $s_{c}^{n}:=\left(\mathrm{d} x_{1}\right)^{2}+\cdots+\left(\mathrm{d} x_{n}\right)^{2}+c^{-1}\left(\mathrm{~d} x_{n+1}\right)^{2}$. The condition $x_{n+1}+\frac{c}{|c|} \geq 0$ in the definition of $\mathbb{F}_{c}^{n}$ guarantees that $\mathbb{F}_{c}^{n}$ is connected also when $c<0$. We denote, as usual, $\mathbb{F}_{1}^{n}$ and $\mathbb{F}_{-1}^{n}$ by $S^{n}$ and $\mathbb{H}^{n}$ respectively.

Remark 2.3 Note that in the definition above there is an underlying continuity with respect to the curvature parameter $c$, once we disregard the connectedness assumption. More precisely, the set

$$
\left\{\left(x_{1}, \ldots, x_{n+1}\right) \in \mathbb{R}^{n+1} \mid c\left(x_{1}^{2}+\cdots+x_{n}^{2}\right)+x_{n+1}^{2}=1\right\}
$$

consists of the two hyperplanes $x_{n+1}= \pm 1$ when $c=0$, a two-sheeted hyperboloid with fixed vertices $(0, \ldots, 0, \pm 1)$ and foci $\left(0, \ldots, 0, \pm \frac{c-1}{c}\right)$ when $c<0$, and an ellipse with vertices $(0, \ldots, 0, \pm 1)$ in the $x_{n+1}$-axis and semiaxes of length $\frac{1}{\sqrt{c}}$ on the hyperplane 
$x_{n+1}=0$. This is in accordance with the definition of the tensor $s_{c}^{n}$ since, for it to behave well when $c \rightarrow 0^{+}$or $c \rightarrow 0^{-}$, one needs to impose $\mathrm{d} x_{n+1}=0$ when $c=0$.

Let $G_{c}(n)$ be the identity component of the Lie group of linear maps $\mathbb{R}^{n+1} \rightarrow \mathbb{R}^{n+1}$ that leave invariant the bilinear form

$$
\langle x, y\rangle_{c}^{n}:=\sum_{i=1}^{n} x_{i} y_{i}+c^{-1} x_{n+1} y_{n+1},
$$

for $x=\left(x_{1}, \ldots, x_{n+1}\right), y=\left(y_{1}, \ldots, y_{n+1}\right)$. Observe that $G_{1}(n)=\mathrm{SO}(n+1)$ and $G_{-1}(n)=\mathrm{SO}_{0}(n, 1)$, the identity component of $\mathrm{O}(n, 1)$.

If $c=0$, the space form $\left(\mathbb{F}_{0}^{n}, \mathbf{g}_{0}^{n}\right)$ is simply equal to $\mathbb{R}^{n}$ with the Euclidean metric, $G_{0}(n)$ is set to be the group $\operatorname{SE}(n)$, the special Euclidean group of $\left(\mathbb{F}_{0}^{n}, \mathbf{g}_{0}^{n}\right)$. Recall that $\mathrm{SE}(n)$ is equal to $\mathbb{R}^{n} \times \mathrm{SO}(n)$ as a set, and is equipped with the group operation $\star$ given by

$$
(v, L) \star(u, K):=(L u+v, L \circ K) .
$$

The natural action, also written as $\star$, of $\mathrm{SO}(n)$ on $\mathbb{R}^{n}$ is given by

$$
(u, K) \star v:=K v+u, \quad(u, K) \in \mathrm{SO}(n), v \in \mathbb{R}^{n} .
$$

Finally recall that, with this notation, the identity component of the isometry group of $\left(\mathbb{F}_{c}^{n}, \mathbf{g}_{c}^{n}\right)$ is equal to $G_{c}(n)$ for all $c \in \mathbb{R}$ (cf. [19]).

2.4. Reduction of the rolling problem. When rolling onto a space form it is possible to reduce the controllability problem to the study of certain holonomy groups. In other words, one can consider the change of the initial state of the system after rolling along loops in the space $\Omega_{x}(M)$ of piecewise $C^{1}$-loops in $M$ based at $x$.

The fundamental feature of rolling onto a space form lies in the fact that there is a $G_{c}(n)$-principal bundle structure for the state space compatible with the distribution $\mathcal{D}_{\mathrm{R}}$, i.e. $\mathcal{D}_{\mathrm{R}}$ is a $G_{c}(n)$-principal bundle connection. This was proved in [12] by using Proposition 2.2, and it is provided below.

Proposition 2.4 (i) The bundle $\pi_{Q, M}: Q \rightarrow M$ is a principal $G_{c}(n)$-bundle with a left action $\mu: G_{c}(n) \times Q \rightarrow Q$ defined for every $q=(x, \hat{x} ; A)$ by

$$
\begin{aligned}
\mu((\hat{y}, C), q) & =(x, C \hat{x}+\hat{y} ; C \circ A), & & \text { if } c=0, \\
\mu(B, q) & =(x, B \hat{x} ; B \circ A), & & \text { if } c \neq 0 .
\end{aligned}
$$

Moreover, the action $\mu$ preserves the distribution $\mathcal{D}_{\mathrm{R}}$ i.e., for any $q \in Q$ and $B \in G_{c}(n),\left.\left(\mu_{B}\right)_{*} \mathcal{D}_{\mathrm{R}}\right|_{q}=\left.\mathcal{D}_{\mathrm{R}}\right|_{\mu(B, q)}$ where $\mu_{B}: Q \rightarrow Q ; q \mapsto \mu(B, q)$.

(ii) For any given $q=(x, \hat{x} ; A) \in Q$, there is a unique subgroup $\mathcal{H}_{q}^{c}$ of $G_{c}(n)$, called the holonomy group of $\mathcal{D}_{\mathrm{R}}$, such that

$$
\mu\left(\mathcal{H}_{q}^{c} \times\{q\}\right)=\mathcal{O}_{\mathcal{D}_{\mathrm{R}}}(q) \cap \pi_{Q, M}^{-1}(x) .
$$

Also, if $q^{\prime}=\left(x, \hat{x}^{\prime} ; A^{\prime}\right) \in Q$ is in the same $\pi_{Q, M^{-}}$fiber as $q$, then $\mathcal{H}_{q}^{c}$ and $\mathcal{H}_{q^{\prime}}^{c}$ are conjugate in $G_{c}(n)$ and all conjugacy classes of $\mathcal{H}_{q}^{c}$ in $G_{c}(n)$ are of the form $\mathcal{H}_{q^{\prime}}^{c}$.

An open problem related to the proposition above asks for the extent to which this result holds. More precisely, given two Riemannian manifolds $M$ and $\hat{M}$ of dimension $n \geq 2$ and the canonical projection $Q=Q(M, \hat{M}) \rightarrow M$, can one give conditions on the manifolds so that there exists a $G$-principal bundle structure for some Lie group $G$ so that the rolling distribution $\mathcal{D}_{\mathrm{R}}$ is $G$-equivariant? For instance, this is true if one of the manifolds is a space form. 
For the case $c=0$, one can take advantage of the semi-direct product structure of $\mathrm{SE}(n)$ by considering the projection of the fiber of an orbit onto $\mathrm{SO}(n)$, which is nothing but the Riemannian holonomy group of $M$. As a result, it is proved in [12] that complete controllability holds if and only if $M$ has full holonomy.

For the case when $c \neq 0$, the problem is more subtle. It was shown in [12] that this principal $G_{c}(n)$-bundle structure implies the existence of a vector bundle connection $\nabla^{c}$ on the vector bundle $\pi_{T M \oplus \mathbb{R}}: T M \oplus \mathbb{R} \rightarrow M$, called the rolling connection, defined as follows: for every $x \in M,\left.X \in T\right|_{x} M,(Y, s) \in \operatorname{VF}(M) \times C^{\infty}(M)$,

$$
\nabla_{X}^{c}(Y, s)=\left(\nabla_{X} Y+s(x) X, X(s)-c g\left(\left.Y\right|_{x}, X\right)\right) .
$$

Here we have canonically identified the space of smooth sections $\Gamma\left(\pi_{T M \oplus \mathbb{R}}\right)$ of $\pi_{T M \oplus \mathbb{R}}$ with $\operatorname{VF}(M) \times C^{\infty}(M)$.

The connection $\nabla^{c}$ is a metric connection with respect to the fiber inner product $h_{c}$ on $T M \oplus \mathbb{R}$ defined by

$$
h_{c}((X, r),(Y, s))=g(X, Y)+c^{-1} r s,
$$

where $X,\left.Y \in T\right|_{x} M, r, s \in \mathbb{R}$. Its holonomy group is denoted by $\mathcal{H}^{c}$.

After a trivial scaling, it is enough to consider only the cases $c= \pm 1$. The use of the rolling connection $\nabla^{c}$ on the vector bundle $T M \oplus \mathbb{R}$ has the advantage that it allows one to prove that complete controllability of the rolling system is equivalent to the fact that $\mathcal{H}^{c}$ equals $\mathrm{SO}(n+1)$ for the spherical case $c=1$, or $\mathrm{SO}_{0}(n, 1)$ for the hyperbolic case $c=-1$.

\section{WARPED PRODUCTS}

In order to present our results, we need some standard material on warped products, as presented for example in [23], as well as means to detect when a manifold can be decomposed as the warping of two (or more) manifolds. To set the terminology, we recall the following definition.

Definition $3.1 \quad$ (i) Let $(N, h),(M, g)$ be Riemannian manifolds and $f \in C^{\infty}(N)$ a non-vanishing function. Then the manifold $N \times M$ equipped with the metric

$$
\left.\left(h \oplus_{f} g\right)\right|_{(y, x)}:=\left.h\right|_{y}+\left.f(y)^{2} g\right|_{x}, \quad(y, x) \in N \times M,
$$

is a Riemannian manifold called the warped product of $(N, h)$ and $(M, g)$ with warping function $f$.

(ii) Let $(N, h),\left(M_{1}, g_{1}\right),\left(M_{2}, g_{2}\right)$ be Riemannian manifolds and $f_{1}, f_{2} \in C^{\infty}(N)$. Denote by $\mathrm{pr}_{1}: N \times M_{1} \rightarrow N$ the canonical projection. Then $\left(N \times M_{1} \times\right.$ $\left.M_{2},\left(h \oplus_{f_{1}} g_{1}\right) \oplus_{\mathrm{pr}_{1}^{*}\left(f_{2}\right)} g_{2}\right)$ is called the doubly warped product of $(N, g),\left(M_{1}, g_{1}\right)$ and $\left(M_{2}, g_{2}\right)$ with warping functions $f_{1}, f_{2}$. We denote its metric simply by $h \oplus_{f_{1}} g_{1} \oplus_{f_{2}} g_{2}$.

Remark 3.2 Note that the metric of the above doubly warped product at $\left(y, x_{1}, x_{2}\right) \in$ $N \times M_{1} \times M_{2}$ has the form

$$
\left.\left(h \oplus_{f_{1}} g_{1} \oplus_{f_{2}} g_{2}\right)\right|_{\left(y, x_{1}, x_{2}\right)}=\left.h\right|_{y}+\left.f_{1}(y)^{2} g_{1}\right|_{x_{1}}+\left.f_{2}(y)^{2} g_{2}\right|_{x_{2}} .
$$

Therefore, it is easy to see that $\left(N \times M_{1} \times M_{2}, h \oplus_{f_{1}} g_{1} \oplus_{f_{2}} g_{2}\right)$ and $\left(N \times M_{2} \times M_{1}, h \oplus_{f_{2}}\right.$ $\left.g_{2} \oplus_{f_{1}} g_{1}\right)$ are isometric.

The main purpose of this section is to introduce a technical result due to Hiepko, (see Theorem 3.4 below) that we use later in order to detect warped products. In order to state this theorem, we need to introduce some terminology. 
Definition 3.3 A submanifold $N$ of a Riemannian manifold $(M, g)$ is said to be:

- umbilical if there is a local section $\nu$ of the normal bundle $T N^{\perp}$ such that the second fundamental form $\mathrm{II}_{N}$ of $N$ has the form

$$
\mathrm{II}_{N}(X, Y)=g(X, Y) \nu, \quad \forall X,\left.Y \in T\right|_{x} N, x \in N ;
$$

- spherical if $(M, g)$ is umbilical and, in addition, the section $\nu$ satisfies

$$
\nabla_{X} \nu \in T N, \quad \forall X \in T N .
$$

The last condition (7) means that $\nu$ is parallel with respect to the normal connection of $N$. With this at hand, the following well-known theorem holds.

Theorem 3.4 ([17]) Let $(M, g)$ be a Riemannian manifold and suppose there is a smooth constant rank distribution $\mathcal{D}$ on $M$ with the following properties:

(i) Both $\mathcal{D}$ and $\mathcal{D}^{\perp}$ are integrable.

(ii) The integral manifolds of $\mathcal{D}^{\perp}$ are totally geodesic.

(iii) The integral manifolds of $\mathcal{D}$ are spherical.

Then $(M, g)$ is locally a warped product. If moreover $(M, g)$ is complete and simply connected, then $(M, g)$ is globally a warped product. Finally, if $f$ is the warping function and $\nu$ is a section of the bundle $\mathcal{D}^{\perp}$ as in Definitions 3.1 and 3.3, then

$$
\nu=-\frac{\nabla f}{f} \text {. }
$$

Remark 3.5 More precisely, as explained in [17] (see Eqs. (11) and (17) there), under the assumptions of the above theorem, every $x \in M$ has a neighbourhood $U$ and integral manifolds $N, N^{\perp}$ through $x$ of $\mathcal{D}, \mathcal{D}^{\perp}$, respectively, such that $U$ is diffeomorphic to $N^{\perp} \times N$ which maps $\left.g\right|_{U}$ to $\left.g\right|_{N^{\perp}} \oplus_{f} h$, where $h$ is a certain metric on $N$. If $(M, g)$ is complete and simply connected, one may take $U=M$.

\section{Presentation of the main Results}

We now present the main global result of the present paper.

Theorem 4.1 Let $(M, g)$ be a complete and simply connected Riemannian manifold. For $c<0$, the rolling holonomy group $\mathcal{H}^{c}$ is reducible if and only if there exists a complete simply connected Riemannian manifold $\left(M_{1}, g_{1}\right)$ such that $(M, g)$ is a warped product either of the form

(WP1): $\left(\mathbb{R} \times M_{1}, \mathrm{~d} s^{2} \oplus_{e^{c s}} g_{1}\right)$, or

(WP2): $\left(\mathbb{F}_{c}^{k} \times M_{1}, \mathbf{g}_{c}^{k} \oplus_{\cosh (\sqrt{-c} d)} g_{1}\right)$, where $1 \leq k \leq n$ and for each $x \in \mathbb{F}_{c}^{k}$, $d(x)$ is the distance between $x$ and an arbitrary fixed point $x_{0} \in \mathbb{F}_{c}^{k}$.

From the previous result one immediately deduces the following characterization of complete controllability of the rolling problem onto the hyperbolic space $\mathbb{H}^{n}$.

Corollary 4.2 Let $(M, g)$ be a complete, oriented and simply connected Riemannian $n$-manifold rolling onto the space form $\left(\mathbb{H}^{n}, \mathbf{g}_{-1}^{n}\right)$ of curvature -1 . Then the associated rolling problem is completely controllable if and only if $(M, g)$ is not isometric to a warped product of the form (WP1) or (WP2).

Proof of Corollary 4.2. With the notations of Theorem 4.1, and according to Subsection 2.4, studying the rolling problem reduces to determining the holonomy group $\mathcal{H}^{-1}$. Assume that $(M, g)$ is of the form (WP1) or (WP2), then $\mathcal{H}^{-1}$ is a proper subgroup of $\mathrm{SO}_{0}(n, 1)$, i.e., the rolling is not controllable according to [12]. On 
the other hand, if $(M, g)$ is not of the form (WP1) or (WP2), then the action of $\mathcal{H}^{-1}$ must be irreducible. Since $M$ is simply connected, $\mathcal{H}^{-1}$ is connected, and thus it is a connected subgroup of $\mathrm{O}(n, 1)$. Therefore it equals $\mathrm{SO}_{0}(n, 1)$, according to $[5,13]$.

4.1. Proof of the main result. The study of reducibility of $\mathcal{H}^{c}$ in the case $c=0$ corresponds to the classical de Rham theorem, and for $c=1$ this was done in [12]. The rest of the present paper is devoted to the proof of Theorem 4.1. By a trivial rescaling argument, we may assume without loss of generality, that $c=-1$. Theorem 4.1 is a consequence of the following two propositions.

Proposition 4.3 With the notation above, assume that the holonomy group $\mathcal{H}^{-1}$ is reducible. Then $M$ is locally of one of the following forms:

(LW1) a warped product $\left(I \times M_{1}, \mathrm{~d} s^{2} \oplus_{e^{-s}} g_{1}\right)$, where $I \subset \mathbb{R}$ is a certain interval, or (LW2) a doubly warped product $\left(I \times M_{2} \times M_{1}\right.$, d $\left.s^{2} \oplus_{\sinh (s)} g_{2} \oplus_{\cosh (s)} g_{1}\right)$, or

(LW3) a warped product $\left(O \times M_{1}, \mathbf{g}_{-1}^{k} \oplus_{\cosh (d(\cdot))} g_{1}\right)$, where $O \subset \mathbb{H}^{k}$ is a normal neighbourhood of some $x_{0} \in O$ and $d$ is the distance function from $x_{0}$ in $\mathbb{H}^{k}$.

Here $\left(M_{1}, g_{1}\right),\left(M_{2}, g_{2}\right)$ are some Riemannian manifolds.

Proposition 4.4 Suppose $(M, g)$ is a doubly warped product of one of the above forms (LW1), (LW2) or (LW3). Then the holonomy group $\mathcal{H}^{-1}$ is reducible.

Remark 4.5 In the previous propositions, it is possible to replace $\left(I \times M_{1}, \mathrm{~d} s^{2} \oplus_{e^{-s}} g_{1}\right)$ by $\left(-I \times M_{1}, \mathrm{~d} s^{2} \oplus_{e^{s}} g_{1}\right)$, since the map $\left(s, x_{1}\right) \mapsto\left(-s, x_{1}\right)$ provides an isometry between them.

Note that both propositions are of local nature. Along the respective arguments, we will provide the necessary modifications to derive the full proof of Theorem 4.1

Before starting with the proofs, we need to introduce some more notations. The metric $h:=h_{-1}$ associated to the bundle $\pi_{T M \oplus \mathbb{R}}: T M \oplus \mathbb{R} \rightarrow M$ is then

$$
h((X, r),(Y, s))=g(X, Y)-r s, \quad(X, r),\left.(Y, s) \in T\right|_{x} M \oplus \mathbb{R} .
$$

Moreover, for any $X, Y \in \operatorname{VF}(M), s \in C^{\infty}(M)$, the linear connection $\nabla^{-1}$ is given by

$$
\nabla_{X}^{-1}(Y, s)=\left(\nabla_{X} Y+s X, X(s)+g(X, Y)\right) .
$$

In particular, if $\gamma$ is a unit speed geodesic on $M$ and $(Y(t), s(t))$ is parallel along $\gamma$, then

$$
\left\{\begin{array}{l}
\nabla_{\dot{\gamma}} Y+s \dot{\gamma}=0, \\
\dot{s}+g(\dot{\gamma}, Y)=0,
\end{array}\right.
$$

and differentiating once more we get

$$
\left\{\begin{array}{l}
\nabla_{\dot{\gamma}} \nabla_{\dot{\gamma}} Y+\dot{s} \dot{\gamma}=0, \\
\ddot{s}+g\left(\dot{\gamma}, \nabla_{\dot{\gamma}} Y\right)=0,
\end{array}\right.
$$

which simplifies to

$$
\left\{\begin{array}{l}
\nabla_{\dot{\gamma}} \nabla_{\dot{\gamma}} Y=g(\dot{\gamma}, Y) \dot{\gamma} \\
\ddot{s}-s=0 .
\end{array}\right.
$$


4.2. Proof of Proposition 4.3. In this section we provide the proofs of the "only if" parts of Proposition 4.3 and of Theorem 4.1. The proofs of the "if" parts of them is postponed to Section 4.3.

Let $(V, h)$ be a Lorentzian vector space. For a vector subspace $W \subset V$ we define

$$
W^{\perp_{h}}=\{v \in V \mid h(v, w)=0, \forall w \in W\},
$$

the $h$-orthogonal space to $W$. We will occasionally use a notation $\|v\|_{h}^{2}:=h(v, v)$, when $v \in V$.

Let $V_{1}$ be a vector subbundle of $T M \oplus \mathbb{R}$ invariant under the holonomy group $\mathcal{H}^{-1}$ of $\nabla^{-1}$ and set $V_{2}=V_{1}^{\perp_{h}}$. This is again invariant under $\mathcal{H}^{-1}$, since $\nabla^{-1}$ is metric with respect to $h$. Since $\operatorname{dim}\left(V_{1} \cap V_{2}\right) \in\{0,1\}$, the argument is divided into two cases.

4.2.1. Case $V_{1} \cap V_{2}=\{0\}$. We have $T M \oplus \mathbb{R}=V_{1} \oplus V_{2}$. For $\alpha=1,2$, define subsets $N_{\alpha}$ of $M$ by

$$
N_{\alpha}=\left\{x \in M\left|(0,1) \in V_{\alpha}\right|_{x}\right\} .
$$

The restrictions of $h$ to $V_{1}$ and $V_{2}$ are both non-degenerate, and since $h$ has signature $(n, 1), h$ is positive definite on one of them, which we assume without loss of generality to be $V_{2}$. Let us assume $\left.h\right|_{V_{2}}$ has signature $(n-m, 0)$, for some $m$ s.t. $0 \leq m<n$. Therefore $h$ is Lorentzian on $V_{1}$, i.e., $\left.h\right|_{V_{1}}$ has signature $(m, 1)$. In particular, $V_{1}$ intersects transversally the light cone. To this end, notice that since $\nabla^{-1}$ is a metric connection, it preserves the signatures of invariant subbundles $V_{1}, V_{2}$ so the above claims are well established.

First we prove that $N_{1}$ is non-empty and $N_{2}$ is empty in the case where $M$ is complete.

Lemma 4.6 One has $N_{2}=\emptyset$ and if $M$ is complete, then $N_{1} \neq \emptyset$.

Proof. The fact that $N_{2}=\emptyset$ is trivial because

$$
h((0,1),(0,1))=\|(0,1)\|_{h}^{2}=-1,
$$

and $h$ is positive definite on $V_{2}$.

Suppose that $M$ is complete and fix $x_{0} \in M$. Since $h$ is Lorentzian on $V_{1}$, there is a $\left.\left(X_{0}, r_{0}\right) \in V_{1}\right|_{x_{0}}$ such that $\left\|\left(X_{0}, r_{0}\right)\right\|_{h}^{2}<0$. By scaling we may assume that $\left\|\left(X_{0}, r_{0}\right)\right\|_{h}^{2}=-1$, i.e., $\left\|X_{0}\right\|_{g}^{2}-r_{0}^{2}=-1$. Moreover, we may assume that $r_{0}>0$. If $X_{0}=0$, then $r_{0}=1$ and $\left.(0,1) \in V_{1}\right|_{x_{0}}$ and we are done. Hence assume that $X_{0} \neq 0$. Let $\gamma$ be a unit speed geodesic with velocity $X_{0} /\left\|X_{0}\right\|_{g}$ and write $(X(t), r(t))$ for the $\nabla^{-1}$-parallel transport of $\left(X_{0}, r_{0}\right)$ along $\gamma$. Since $r(0)=r_{0}$ and $\dot{r}(0)=-g\left(\dot{\gamma}(0), X_{0}\right)=-\left\|X_{0}\right\|_{g}$, and because $\ddot{r}-r=0$, we get

$$
r(t)=r_{0} \cosh (t)-\left\|X_{0}\right\|_{g} \sinh (t) .
$$

Since $r_{0}^{2}-\left\|X_{0}\right\|_{g}^{2}=1$ and $r_{0}>0$, there exists a unique $t_{1} \in \mathbb{R}$ such that

$$
\left(\cosh \left(t_{1}\right), \sinh \left(t_{1}\right)\right)=\left(r_{0},\left\|X_{0}\right\|_{g}\right) .
$$

Hence $r\left(t_{1}\right)=r_{0}^{2}-\left\|X_{0}\right\|_{g}^{2}=1$. But then

$$
\left\|X\left(t_{1}\right)\right\|_{g}^{2}-1=\left\|X\left(t_{1}\right)\right\|_{g}^{2}-r\left(t_{1}\right)^{2}=\left\|\left(X\left(t_{1}\right), r\left(t_{1}\right)\right)\right\|_{h}^{2}=\left\|\left(X_{0}, r_{0}\right)\right\|_{h}^{2}=-1,
$$

which implies that $\left\|X\left(t_{1}\right)\right\|_{g}^{2}=0$ and hence $(0,1)=\left.\left(X\left(t_{1}\right), r\left(t_{1}\right)\right) \in V_{1}\right|_{\gamma\left(t_{1}\right)}$ i.e. $\gamma\left(t_{1}\right) \in N_{1}$. This finishes the proof. 
For $\alpha=1,2$, let $\pi_{V_{\alpha}}:=\left.\pi_{T M \oplus \mathbb{R}}\right|_{V_{\alpha}}: V_{\alpha} \rightarrow M$ and define smooth sections $\left(W_{\alpha}, w_{\alpha}\right) \in \Gamma\left(\pi_{V_{\alpha}}\right)$, such that at every point $x \in M$,

$$
(0,1)=\left(W_{1}, w_{1}\right)+\left(W_{2}, w_{2}\right) .
$$

Clearly then $W_{1}=-W_{2}, \quad w_{1}+w_{2}=1$. The fact $N_{2}=\emptyset$ means that $w_{1}$ never vanishes on $M$. Indeed, if $w_{1}=0$ at some point, then $w_{2}=1$ and

$$
-1=\|(0,1)\|_{h}^{2}=\left\|\left(W_{1}, 0\right)\right\|_{h}^{2}+\left\|\left(W_{2}, 1\right)\right\|_{h}^{2}=\left\|W_{1}\right\|_{g}^{2}+\left\|W_{2}\right\|_{g}^{2}-1,
$$

hence $W_{1}=0, W_{2}=0$ and $V_{2} \ni\left(W_{2}, w_{2}\right)=(0,1)$, a contradiction.

A simple calculation shows that the curvature $R^{\nabla^{-1}}$ of the rolling connection $\nabla^{-1}$ is given by

$$
R^{\nabla^{-1}}((X, r),(Y, s))(Z, u)=(R(X, Y) Z+B(X, Y) Z, 0)
$$

where $B(X, Y) Z:=g(Y, Z) X-g(X, Z) Y$.

Lemma 4.7 For all $x \in M$ and $X,\left.Y \in T\right|_{x} M$, one has

$$
R(X, Y) W_{\alpha}=-B(X, Y) W_{\alpha}, \quad \alpha=1,2 .
$$

Proof. Notice that for any $(X, r),\left.(Y, s) \in T\right|_{x} M \oplus \mathbb{R}$ one has

$$
R^{\nabla^{-1}}((X, r),(Y, s))(0,1)=(R(X, Y) 0+B(X, Y) 0,0)=(0,0) .
$$

On the other hand, if $\left.\mathfrak{h}^{-1}\right|_{x}$ denotes the Lie algebra of $\left.\mathcal{H}^{-1}\right|_{x}$, by the Ambrose-Singer theorem $\left.R^{\nabla^{-1}}((X, r),(Y, s)) \in \mathfrak{h}^{-1}\right|_{x}$, so

$$
\left.\left.R^{\nabla^{-1}}((X, r),(Y, s)) V_{\alpha}\right|_{x} \subset V_{\alpha}\right|_{x}, \quad \alpha=1,2 .
$$

Hence

$$
\begin{aligned}
(0,0) & =R^{\nabla^{-1}}((X, r),(Y, s))(0,1) \\
& =\underbrace{R^{\nabla^{-1}}((X, r),(Y, s))\left(W_{1}, w_{1}\right)}_{\in V_{1}}+\underbrace{R^{\nabla^{-1}}((X, r),(Y, s))\left(W_{2}, w_{2}\right)}_{\in V_{2}},
\end{aligned}
$$

because $\left(W_{\alpha}, w_{\alpha}\right) \in V_{\alpha}, \alpha=1,2$. Therefore, since $V_{1} \cap V_{2}=\{0\}$, we have

$$
R^{\nabla^{-1}}((X, r),(Y, s))\left(W_{\alpha}, w_{\alpha}\right)=(0,0), \quad \alpha=1,2,
$$

which means that

$$
R(X, Y) W_{\alpha}+B(X, Y) W_{\alpha}=0, \quad \alpha=1,2,
$$

and hence the claim has been established.

Define for every $x \in M$,

$$
\left.V_{\alpha}^{M}\right|_{x}:=\left.\left\{X \mid(X, r) \in V_{\alpha}\right\} \subset T\right|_{x} M, \quad \alpha=1,2 .
$$

Clearly $V_{\alpha}^{M}$ is a smooth distribution on $M \backslash N_{\alpha}$ with $\operatorname{rank} V_{\alpha}^{M}=\operatorname{rank} V_{\alpha}$. In particular, $V_{2}^{M}$ is a smooth constant rank distribution on all of $M$, since $N_{2}=\emptyset$. Moreover, it is clear that $V_{1}^{M}$ is a smooth non-constant rank distribution and that $\operatorname{rank} V_{1}^{M}=\operatorname{rank} V_{1}-1=m$ at points $x \in N_{1}$.

Lemma 4.8 For every $x \in M$, the intersection $V_{1}^{M} \cap V_{2}^{M}$ is spanned by $W_{1}\left(=-W_{2}\right)$ and so is one dimensional on $M \backslash N_{1}$ and zero on $N_{1}$. 
Proof. Indeed, if $X \in V_{1}^{M} \cap V_{2}^{M}$, then there are $r_{1}, r_{2} \in \mathbb{R}$ such that $\left(X, r_{\alpha}\right) \in V_{\alpha}$, $\alpha=1,2$. But then one has

$$
\begin{aligned}
\underbrace{\left(X, r_{1}\right)}_{\in V_{1}}-\underbrace{\left(X, r_{2}\right)}_{\in V_{2}} & =\left(0, r_{1}-r_{2}\right)=\left(r_{1}-r_{2}\right)(0,1) \\
& =\left(r_{1}-r_{2}\right) \underbrace{\left(W_{1}, w_{1}\right)}_{\in V_{1}}+\left(r_{1}-r_{2}\right) \underbrace{\left(W_{2}, w_{2}\right)}_{\in V_{2}},
\end{aligned}
$$

and since $V_{1} \cap V_{2}=\{0\}$, one has

$$
\begin{aligned}
& \left(r_{1}-r_{2}\right)\left(W_{1}, w_{1}\right)=\left(X, r_{1}\right), \\
& \left(r_{2}-r_{1}\right)\left(W_{2}, w_{2}\right)=\left(X, r_{2}\right) .
\end{aligned}
$$

In particular, $X=\left(r_{1}-r_{2}\right) W_{1}$, which shows that $V_{1}^{M} \cap V_{2}^{M} \subset \mathbb{R} W_{1}$. Finally, since $W_{1} \in V_{1}^{M}, W_{2} \in V_{2}^{M}$ and $W_{1}=-W_{2}$, we have that $\mathbb{R} W_{1} \subset V_{1}^{M} \cap V_{2}^{M}$.

Define $\mathcal{D}_{1}:=\left(V_{2}^{M}\right)^{\perp}$ and $\mathcal{D}_{2}:=\left(V_{1}^{M}\right)^{\perp}$ i.e. the orthogonal complements of $V_{2}$ and $V_{1}$ with respect to $g$. Notice that $\mathcal{D}_{\alpha} \subset V_{\alpha}$ for $\alpha=1,2$. Since $V_{2}^{M}$ is a smooth constant rank distribution on $M$ then so is $\mathcal{D}_{1}$ as well and rank $\mathcal{D}_{1}=m$. Similarly, $\mathcal{D}_{2}$ has constant rank $n-m-1$ on $M \backslash N_{1}$ and rank $n-m$ on $N_{1}$. It is obvious that $\mathcal{D}_{2}$ is a smooth distribution on $M \backslash N_{1}$. However, it is not continuous at points of $x \in N_{1}$. Indeed, as will be proved in Lemma 4.9 below, $N_{1}$ is a submanifold of $M$ positive codimension (it has dimension $m$ ). But the rank of a continuous distribution is lower semicontinuous and hence can only locally increase, while $\mathcal{D}_{2}$ has rank $n-m$ on the nowhere dense set $N_{1}$ which is higher than its rank $n-m-1$ on $M \backslash N_{1}$, so $\mathcal{D}_{2}$ cannot be continuous at points of $N_{1}$.

Lemma 4.9 Let $\{\alpha, \beta\}=\{1,2\}$. The distribution $\mathcal{D}_{\alpha}$ is integrable on $M \backslash N_{\beta}$ and the set $N_{1}$ is an integral manifold of $\mathcal{D}_{1}$ which is embedded in $M$. Moreover, if $O$ is an integral manifold of $\mathcal{D}_{\alpha}$ and if $\mathrm{II}_{O}$ is its second fundamental form, then for every $X,\left.Y \in T\right|_{x} O, x \in M \backslash N_{\beta}$,

$$
\mathrm{II}_{O}(X, Y)=\frac{g(X, Y)}{w_{\alpha}} W_{\alpha}
$$

In particular, each integral manifold of $\mathcal{D}_{\alpha}$ is umbilical and $N_{1}$ is totally geodesic.

Proof. Recall that $\mathcal{D}_{\alpha}=\left(V_{\beta}^{M}\right)^{\perp}$ and $T M \oplus \mathbb{R}=V_{\alpha} \oplus V_{\beta}$. Suppose $X, Y$ are vector fields tangent to $\mathcal{D}_{\alpha}$ on $M \backslash N_{\beta}$. Then

$$
\{0\}=g\left(\{Y\} \times V_{\beta}^{M}\right)=h\left(\{(Y, 0)\} \times V_{\beta}\right),
$$

so $(Y, 0) \in V_{\alpha}$, and similarly $(X, 0) \in V_{\alpha}$. Hence

$$
V_{\alpha} \ni \nabla_{X}^{-1}(Y, 0)=\left(\nabla_{X} Y, g(X, Y)\right) .
$$

Similarly, $\left(\nabla_{Y} X, g(Y, X)\right) \in V_{\alpha}$ and thus

$$
([X, Y], 0)=\left(\nabla_{X} Y, g(X, Y)\right)-\left(\nabla_{Y} X, g(Y, X)\right) \in V_{\alpha} .
$$

Therefore

$$
g\left(\{[X, Y]\} \times V_{\beta}^{M}\right)=h\left(\{([X, Y], 0)\} \times V_{\beta}\right)=\{0\},
$$

so $[X, Y]$ is tangent to $\left(V_{\beta}^{M}\right)^{\perp}=\mathcal{D}_{\alpha}$. This proves that $\mathcal{D}_{\alpha}$ is involutive and hence integrable on $M \backslash N_{\beta}$. 
Let $O$ be an integral manifold of $\mathcal{D}_{\alpha}$ in $M \backslash N_{\beta}$ and let $X, Y$ be tangent to $O$. By what we have shown above,

$$
\begin{aligned}
V_{\alpha} \ni \nabla_{X}^{-1}(Y, 0) & =\left(\nabla_{X} Y, g(X, Y)\right)=\left(\nabla_{X} Y, 0\right)+g(X, Y)(0,1) \\
& =\left(\nabla_{X} Y, 0\right)+g(X, Y)\left(W_{\alpha}, w_{\alpha}\right)+g(X, Y)\left(W_{\beta}, w_{\beta}\right) \\
& =\left(\nabla_{X} Y+g(X, Y) W_{\beta}, g(X, Y) w_{\beta}\right)+g(X, Y)\left(W_{\alpha}, w_{\alpha}\right),
\end{aligned}
$$

and so

$$
\left(\nabla_{X} Y+g(X, Y) W_{\beta}, g(X, Y) w_{\beta}\right) \in V_{\alpha} .
$$

Since also $w_{\beta}\left(\nabla_{X} Y, g(X, Y)\right) \in V_{\alpha}$, it follows that

$$
\left(\left(1-w_{\beta}\right) \nabla_{X} Y+g(X, Y) W_{\beta}, 0\right) \in V_{\alpha},
$$

and hence, because $1-w_{\beta}=w_{\alpha}$ and $W_{\beta}=-W_{\alpha}$,

$$
0=h\left(\left\{\left(w_{\alpha} \nabla_{X} Y-g(X, Y) W_{\alpha}, 0\right)\right\} \times V_{\beta}\right)=g\left(\left\{w_{\alpha} \nabla_{X} Y-g(X, Y) W_{\alpha}\right\} \times V_{\beta}^{M}\right) .
$$

Thus $w_{\alpha} \nabla_{X} Y-g(X, Y) W_{\alpha} \in \mathcal{D}_{\alpha}$. Since $W_{\alpha}=-W_{\beta} \in V_{\beta}^{M}=\mathcal{D}_{\alpha}^{\perp}$, this proves that

$$
\mathrm{II}_{O}(X, Y)=\frac{g(X, Y)}{w_{\alpha}} W_{\alpha} .
$$

We show that $N_{1}$ is an integral manifold of $\mathcal{D}_{1}$. Indeed, let $x_{1} \in N_{1}$ and let $\left(Y_{i}, s_{i}\right), i=1, \ldots, n-m$, be a local basis of $V_{2}$ on an open set $U \ni x_{1}$. Since $h$ is positive definite on $V_{2}$, we may assume that the basis $\left(Y_{i}, s_{i}\right), i=1, \ldots, n-m$ is $h$ orthonormal. Moreover, if $x \in N_{1}$, then for all $i, s_{i}(x)=-h\left((0,1),\left(\left.Y_{i}\right|_{x}, s_{i}(x)\right)\right)=0$ since $\left.(0,1) \in V_{1}\right|_{x}$.

Define $F: U \rightarrow \mathbb{R}^{n-m}$ by

$$
F=\left(h\left(\left(Y_{1}, s_{1}\right),(0,1)\right), \ldots, h\left(\left(Y_{n-m}, s_{n-m}\right),(0,1)\right)\right),
$$

and notice that $F^{-1}(0)=N_{1} \cap U$. To show that $N_{1}$ is a smooth embedded submanifold of dimension $m$, it thus suffices to show that $F$ is a submersion at every point $x \in N_{1} \cap U$. But if $x \in N_{1} \cap U$ and $k=1, \ldots, n-m$, then

$$
\left.F_{*}\right|_{x}\left(Y_{k}\right)=\left(h\left(\nabla_{\left.Y_{k}\right|_{x}}^{-1}\left(Y_{i}, s_{i}\right),(0,1)\right)+h\left(\left(\left.Y_{i}\right|_{x}, s_{k}(x)\right), \nabla_{Y_{k}}^{-1}(0,1)\right)\right)_{i=1}^{n-m},
$$

because $\nabla^{-1}$ is metric w.r.t. $h$. Since $\left.\nabla_{\left.Y_{k}\right|_{x}}^{-1}\left(Y_{i}, s_{i}\right) \in V_{2}\right|_{x}$, and $\left.(0,1) \in V_{1}\right|_{x}$, the term $h\left(\nabla_{\left.Y_{k}\right|_{x}}^{-1}\left(Y_{i}, s_{i}\right),(0,1)\right)$ vanishes. Moreover

$$
\begin{aligned}
h\left(\left(\left.Y_{i}\right|_{x}, s_{k}(x)\right), \nabla_{Y_{k}}^{-1}(0,1)\right) & =h\left(\left(\left.Y_{i}\right|_{x}, s_{i}(x)\right),\left(\left.Y_{k}\right|_{x}, 0\right)\right) \\
& =h\left(\left(\left.Y_{i}\right|_{x}, s_{i}(s)\right),\left(\left.Y_{k}\right|_{x}, s_{k}(x)\right)\right)=\delta_{i k},
\end{aligned}
$$

since $s_{k}(x)=0$. Hence if $e_{i}, i=1, \ldots, n-m$, is the canonical basis of $\mathbb{R}^{n-m}$, then for all $x \in N_{1} \cap U,\left.F_{*}\right|_{x}\left(Y_{k}\right)=e_{k}, k=1, \ldots, n-m$ and so they are linearly independent. Hence $F$ is submersive at every point of $N_{1} \cap U$.

To show that $\left.T\right|_{x} N_{1}=\left.\mathcal{D}_{1}\right|_{x}$ for all $x \in U \cap N_{1}$, notice that if $\left.X \in \mathcal{D}_{1}\right|_{x}$, then by computation as above,

$$
\left.F_{*}\right|_{x}(X)=\left(h\left(\nabla_{X}^{-1}\left(Y_{i}, s_{i}\right),(0,1)\right)+g\left(\left.Y_{i}\right|_{x}, X\right)\right)_{i=1}^{n-m}=0,
$$

because $\left.\nabla_{X}^{-1}\left(Y_{i}, s_{i}\right) \in V_{2}\right|_{x},\left.(0,1) \in V_{1}\right|_{x}$ and $\left.\left.Y_{i}\right|_{x} \in V_{2}^{M}\right|_{x}$ while $\left.X \in \mathcal{D}_{1}\right|_{x}=$ $\left(\left.V_{2}^{M}\right|_{x}\right)^{\perp}$. This shows that $\left.\left.\mathcal{D}_{1}\right|_{x} \subset T\right|_{x} N_{1}$ for all $x \in N_{1} \cap U$ and since both linear spaces have dimension $m$, we have the equality i.e. $N_{1}$ is an integral manifold of $\mathcal{D}_{1}$. 
Finally, since $N_{1}$ is an integral manifold of $\mathcal{D}_{1}$ and since $\left(W_{1}, w_{1}\right)=(0,1)$ on $N_{1}$, one has that the second fundamental form $\mathrm{II}_{N_{1}}$ vanishes on $N_{1}$. Therefore $N_{1}$ is totally geodesic.

In particular, at every $x \in N_{1}$ one has $\left.V_{1}^{M}\right|_{x}=\left.\mathcal{D}_{1}\right|_{x}=\left.T\right|_{x} N_{1}$.

Lemma 4.10 Let $\{\alpha, \beta\}=\{1,2\}$. The integral manifolds of $\mathcal{D}_{\alpha}$ in $M \backslash N_{\beta}$ are spherical.

Proof. We need to show that $\nabla_{X}\left(w_{\alpha}^{-1} W_{\alpha}\right) \in \mathcal{D}_{\alpha}$ for all $X \in \mathcal{D}_{\alpha}$ on $M \backslash N_{\beta}$. Indeed, $g\left(W_{\alpha}, X\right)=0$ because $W_{\alpha}=-W_{\beta} \in V_{\beta}^{M}=\mathcal{D}_{\alpha}^{\perp}$ and since $\left(w_{\alpha}^{-1} W_{\alpha}, 1\right)=$ $w_{\alpha}^{-1}\left(W_{\alpha}, w_{\alpha}\right) \in V_{\alpha}$, we have

$$
\begin{aligned}
V_{\alpha} \ni \nabla_{X}^{-1}\left(w_{\alpha}^{-1} W_{\alpha}, 1\right) & =\left(\nabla_{X}\left(w_{\alpha}^{-1} W_{\alpha}\right)+X, 0+g\left(X, w_{\alpha}^{-1} W_{\alpha}\right)\right) \\
& =\left(\nabla_{X}\left(w_{\alpha}^{-1} W_{\alpha}\right)+X, 0\right) .
\end{aligned}
$$

Because $X \in\left(V_{\beta}^{M}\right)^{\perp}$, it then follows that

$$
\begin{aligned}
0 & \left.=h\left(\left\{\left(\nabla_{X}\left(w_{\alpha}^{-1} W_{\alpha}\right)+X, 0\right)\right\} \times V_{\beta}\right)=g\left(\left\{\nabla_{X}\left(w_{\alpha}^{-1} W_{\alpha}\right)+X\right)\right\} \times V_{\beta}^{M}\right) \\
& =g\left(\left\{\nabla_{X}\left(w_{\alpha}^{-1} W_{\alpha}\right)\right\} \times V_{\beta}^{M}\right),
\end{aligned}
$$

i.e., $\nabla_{X}\left(w_{\alpha}^{-1} W_{\alpha}\right) \in\left(V_{\beta}^{M}\right)^{\perp}=\mathcal{D}_{\alpha}$.

Lemma 4.11 The distributions $V_{1}^{M}$ and $V_{2}^{M}$ are integrable and their integral manifolds are totally geodesic.

Proof. Fix $\alpha=1,2$ and let $x \in M \backslash N_{\alpha}$. Since $V_{\alpha}^{M}$ has constant rank around $x$, the integrability of it in a neighborhood $U$ of $x$ which does not intersect $N_{\alpha}$, is equivalent to the involutivity of $V_{\alpha}^{M}$ on $U$.

Thus take $X, Y \in \operatorname{VF}(U)$ which are tangent to $V_{\alpha}^{M}$. Then there is a unique $s \in C^{\infty}(U)$ such that $(Y, s) \in V_{\alpha}$ on $U$. But then

$$
V_{\alpha} \ni \nabla_{X}^{-1}(Y, s)=\left(\nabla_{X} Y+s X, X(s)+g(X, Y)\right),
$$

which implies that on $U$

$$
\nabla_{X} Y+s X \in V_{\alpha}^{M}
$$

Since $X$ is also tangent to $V_{\alpha}^{M}$ on $U$, we get that $\nabla_{X} Y \in V_{\alpha}^{M}$ on $U$ as well.

But since $\nabla$ is torsion free and since by the above $\nabla_{X} Y, \nabla_{Y} X \in V_{\alpha}^{M}$ on $U$, one has that $[X, Y]=\nabla_{X} Y-\nabla_{Y} X \in V_{\alpha}^{M}$ on $U$, i.e. $V_{\alpha}^{M}$ is involutive on $U$.

Moreover, if $O$ is an integral manifold of $V_{\alpha}^{M}$ through $y \in U$, and if $X, Y \in \operatorname{VF}(O)$, then on some neighborhood $U^{\prime} \subset U$ of $y$ in $M$, there are $\tilde{X}, \tilde{Y} \in \operatorname{VF}\left(U^{\prime}\right)$ which restrict to $X, Y$ on $O$ and are tangent to $V_{\alpha}^{M}$ on $U^{\prime}$. Then $\nabla_{X} Y=\nabla_{\tilde{X}} \tilde{Y}$ on $O$ and by what was shown above, this is tangent to $V_{\alpha}^{M}$ i.e. tangent to $O$. Thus $O$ is totally geodesic.

This proves that $V_{\alpha}^{M}$ is involutive on $M \backslash N_{\alpha}$ and that its integral manifolds are totally geodesic. Since $N_{2}=\emptyset$, the only thing left is to notice that by Lemma 4.9, $N_{1}$ is an integral manifold of $V_{1}^{M}$ because $\left.\mathcal{D}_{1}\right|_{y}=\left.V_{1}^{M}\right|_{y}$ for all $y \in N_{1}$.

Lemma 4.12 For every $x \in M$ and every unit vector $\left.u \in T\right|_{x} M$, one has

$$
\left(P^{\nabla^{-1}}\right)_{0}^{t}\left(\gamma_{u}\right)(0,1)=\left(-\sinh (t) \dot{\gamma}_{u}(t), \cosh (t)\right)
$$

where $\gamma_{u}(t)=\exp _{x}(t u)$. In particular, if $x \in N_{1}$ and $\left.u \in V_{2}^{M}\right|_{x},\|u\|_{g}=1$, then $\dot{\gamma}_{u}(t) \in V_{1}^{M} \cap V_{2}^{M}$ for all $t \neq 0$. 
Proof. Let $(X(t), r(t)):=\left(-\sinh (t) \dot{\gamma}_{u}(t), \cosh (t)\right)$. Then $(X(0), r(0))=(0,1)$ and

$$
\begin{aligned}
& \nabla_{\dot{\gamma}_{u}(t)}^{-1}(X, r) \\
= & \left(\nabla_{\dot{\gamma}_{u}(t)}\left(-\sinh (t) \dot{\gamma}_{u}(t)\right)+\cosh (t) \dot{\gamma}_{u}(t), \frac{\mathrm{d}}{\mathrm{d} t} \cosh (t)+g\left(-\sinh (t) \dot{\gamma}_{u}(t), \dot{\gamma}_{u}(t)\right)\right. \\
= & \left(-\cosh (t) \dot{\gamma}_{u}(t)+\cosh (t) \dot{\gamma}_{u}(t), \sinh (t)-\sinh (t) g(u, u)\right)=(0,0),
\end{aligned}
$$

where at the last equality we used that $\|u\|_{g}=1$. This proves that

$$
(X(t), r(t))=\left(P^{\nabla^{-1}}\right)_{0}^{t}\left(\gamma_{u}\right)(0,1)
$$

We prove the second claim. Let $x \in N_{1}$ and $\left.u \in V_{2}^{M}\right|_{x},\|u\|_{g}=1$. Since $V_{2}^{M}$ is integrable and its integral manifolds are totally geodesic by Lemma 4.11, it follows that $\dot{\gamma}_{u}(t) \in V_{2}^{M}$ for all $t$. On the other hand, since $\left.(0,1) \in V_{1}\right|_{x}$ by the definition of the set $N_{1}$, we have $\left(P^{\nabla^{-1}}\right)_{0}^{t}\left(\gamma_{u}\right)(0,1) \in V_{1}$ for all $t$ i.e. $\left(-\sinh (t) \dot{\gamma}_{u}(t), \cosh (t)\right) \in V_{1}$ for all $t$ and this implies that $-\sinh (t) \dot{\gamma}_{u}(t) \in V_{1}^{M}$ for all $t$. Hence $\dot{\gamma}_{u}(t) \in V_{1}^{M}$ if $t \neq 0$.

Lemma 4.13 (i) Let $\{\alpha, \beta\}=\{1,2\}$. Then if $x \notin M \backslash N_{\beta}$, then there is an integral manifold $O_{\alpha}$ of $V_{\alpha}^{M}$ through $x$ such that $\left(O_{\alpha},\left.g\right|_{O_{\alpha}}\right)$ is isometric to $\left(I \times M_{\alpha}, \mathrm{d} s^{2} \oplus_{f_{\alpha}(s)} g_{\alpha}\right)$ where $I \subset \mathbb{R}$ is an open interval and $f_{\alpha} \in C^{\infty}(I)$ satisfies $f_{\alpha}^{\prime \prime}-f_{\alpha}=0$.

(ii) If $x \in N_{1}$ then there exists an integral manifold $O_{2}$ of $V_{2}^{M}$ through $x$ and $\left(\mathrm{O}_{2},\left.g\right|_{\mathrm{O}_{2}}\right)$ has constant curvature -1 if $\operatorname{rank} V_{2}^{M} \geq 2$.

Proof. (i) Without loss of generality, one may assume that $\alpha=2, \beta=1$, since the proof of the other case is completely symmetric. Assume that $x \notin N_{1}$ and let $O_{2}$ be an integral manifold of $V_{2}^{M}$ through $x \in M$ such that $O_{2} \cap N_{1}=\emptyset$. Clearly one can assume that $\operatorname{dim} \mathrm{O}_{2} \geq 2$. In this case, the 1-dimensional integral manifolds of the distribution $\mathbb{R} W_{2}=V_{1}^{M} \cap V_{2}^{M}$ spanned by $W_{2}$ on $O_{2}$ are geodesics since they are (locally) the intersections of integral manifolds of $V_{1}^{M}$ and $V_{2}^{M}$, which are totally geodesic by Lemma 4.11. Moreover, integral manifolds of $\mathcal{D}_{2}$ are spherical by Lemma 4.9 and $\left.T\right|_{y} O_{2}=\left.\left.\mathbb{R} W_{2}\right|_{y} \oplus \mathcal{D}_{2}\right|_{y}$ for all $y \in O_{2}$, so $O_{2}$ is locally a warped product (see Theorem 3.4) of the form $\left(I \times M_{2}, \mathrm{~d} s^{2} \oplus_{f_{2}(s)} g_{2}\right)$ where $I \subset \mathbb{R}$ is an open interval and $f_{2} \in C^{\infty}(I)$. Moreover, for $y \in O_{2}$ and $\left.X \in \mathcal{D}_{2}\right|_{y}=\left.T\right|_{y} M_{2}$, we have

$g\left(W_{2}, W_{2}\right) X=B\left(X, W_{2}\right) W_{2}=-R\left(X, W_{2}\right) W_{2}=\frac{H^{f_{2}}\left(W_{2}, W_{2}\right)}{f_{2}} X=\frac{f_{2}^{\prime \prime}}{f_{2}} g\left(W_{2}, W_{2}\right) X$

where the second equality follows from Lemma 4.7 and third equality follows from [23], Proposition 42, case (2) (here $H^{f_{2}}$ is the Hessian of $f_{2}$ ). Taking any non-zero $\left.X \in \mathcal{D}_{2}\right|_{y}$ (there exist one since $\operatorname{rank} \mathcal{D}_{2} \geq 1$ ) and noticing that $\left.W_{2}\right|_{y} \neq 0$ since $y \notin N_{1}$, we get the claimed equation $f_{2}^{\prime \prime}=f_{2}$ for $f_{2}$. This establishes the first part of the lemma.

(ii) Assume that $x \in N_{1}$. Let $k:=\operatorname{rank} V_{2}^{M}=n-m$ and let $\epsilon>0$ be small enough such that $\exp _{x}$ is a diffeomorphism from $B:=\left\{\left.X \in V_{2}^{M}\right|_{x} \mid\|X\|_{g}<\epsilon\right\}$ onto its image and so that $B \cap N_{1}=\{x\}$ (this is possible since $N_{1}$ is embedded in $M$ ). Since integral manifolds of $V_{2}^{M}$ are totally geodesic, it follows that $O_{2}:=\exp _{x} B$ is an integral manifold of $V_{2}^{M}$. 
Suppose that $u,\left.X \in V_{2}^{M}\right|_{x}$ are such that $u \perp X$ and $\|u\|_{g}=1$ and define for $t \in[0, \epsilon[$

$$
\begin{aligned}
\gamma_{u}(t) & :=\exp _{x}(t u), \\
Y_{u, X}(t) & :=\sinh (t) P_{0}^{t}\left(\gamma_{u}\right) X .
\end{aligned}
$$

We claim that $Y_{u, X}$ is the Jacobi field $Y$ along $\gamma_{u}$ such that $Y(0)=0, \nabla_{u} Y(0)=X$.

The claims about initial values being obviously true for $Y_{u, X}$, it remains to show that $Y_{u, X}$ satisfies the Jacobi-equation. Notice that $\dot{\gamma}_{u}(t) \perp Y_{u, X}(t)$ for all $t$ and recall that by Lemmas 4.8 and $4.12, \dot{\gamma}_{u}(t) \in V_{1}^{M} \cap V_{2}^{M}=\mathbb{R} W_{1}$ when $t \neq 0$. Therefore Lemma 4.7 implies that

$$
R\left(\dot{\gamma}_{u}(t), Y_{u, X}(t)\right) \dot{\gamma}_{u}(t)=-B\left(\dot{\gamma}_{u}(t), Y_{u, X}(t)\right) \dot{\gamma}_{u}(t)=g\left(\dot{\gamma}_{u}(t), \dot{\gamma}_{u}(t)\right) Y_{u, X}(t)=Y_{u, X}(t),
$$

while

$$
\nabla_{\dot{\gamma}_{u}(t)} \nabla_{\dot{\gamma}_{u}} Y_{u, X}(t)=\nabla_{\dot{\gamma}_{u}(t)}\left(\cosh (t) P_{0}^{t}\left(\gamma_{u}\right) X\right)=\sinh (t) P_{0}^{t}\left(\gamma_{u}\right) X=Y_{u, X}(t) .
$$

Hence the claim has been established.

Let $S^{k-1}$ be the $(k-1)$-dimensional unit sphere $\left\{\left.X \in V_{2}^{M}\right|_{x} \mid\|X\|_{g}=1\right\}$ of $\left.V_{2}^{M}\right|_{x}$ and define

$$
F:(] 0, \epsilon\left[\times S^{k-1},\left.\mathrm{~d} s^{2} \oplus_{\sinh (s)} g\right|_{S^{k-1}}\right) \rightarrow(M, g) ; \quad F(s, X)=\exp _{x}(s X) .
$$

Then $F$ is a diffeomorphism onto $O_{2} \backslash\{x\}=\exp _{x}(B \backslash\{0\})$ and

$$
\left.F_{*}\right|_{(s, u)}\left(\alpha \partial_{s}+X\right)=\alpha \dot{\gamma}_{u}(s)+Y_{u, X}(s),
$$

whence

$$
\begin{aligned}
\left\|\left.F_{*}\right|_{(s, u)}\left(\alpha \partial_{s}+X\right)\right\|_{g}^{2} & =\alpha^{2}\|u\|_{g}^{2}+\left\|Y_{u, X}(s)\right\|_{g}^{2}=\alpha^{2}+\sinh ^{2}(s)\|X\|_{g}^{2} \\
& =\left\|\alpha \partial_{s}+X\right\|_{\left.\mathrm{d} s^{2} \oplus_{\sinh (s)} g\right|_{S^{k-1}}}^{2} .
\end{aligned}
$$

This means that the mapping $F$ is an isometry from (] $0, \epsilon\left[\times S^{k-1},\left.\mathrm{~d} s^{2} \oplus_{\sinh (s)} g\right|_{S^{k-1}}\right)$ onto $\left(O_{2} \backslash\{x\},\left.g\right|_{O_{2} \backslash\{x\}}\right)$. Since the former Riemannian manifold has constant curvature -1 , if $k \geq 2$, it follows that $\left(O_{2},\left.g\right|_{O_{2}}\right)$ has constant curvature -1 , if $k \geq 2$.

We now finish the proof of Proposition 4.3.

Proof of Proposition 4.3. First we consider the case where $k:=\operatorname{rank} V_{2}^{M} \geq 2$. Let $x \in M$ be fixed. Since integral manifolds of the constant rank distribution $\mathcal{D}_{1}$ are spherical and those of $V_{2}^{M}=\mathcal{D}_{1}^{\perp}$ are totally geodesic, Theorem 3.4 implies that there is a neighborhood $U$ of $x$ in $M$ such that $\left(U,\left.g\right|_{U}\right)$ is isometric to a warped product $\left(O_{2} \times M_{1},\left.g\right|_{O_{2}} \oplus_{f_{1}} g_{1}\right)$ where $O_{2}$ is an integral manifold of $V_{2}^{M}$ through $x$, $M_{1}$ is an integral manifold of $\mathcal{D}_{1}$ through $x$ and $f_{1} \in C^{\infty}\left(O_{2}\right)$ (see Remark 3.5).

First consider the situation where $x \in M \backslash N_{1}$. By Lemma 4.13, after shrinking $U$ if necessary around $x,\left(O_{2},\left.g\right|_{O_{2}}\right)$ is isometric to $\left(I \times M_{2}\right.$, $\left.\mathrm{d} s^{2} \oplus_{f_{2}(s)} g_{2}\right)$ where $I \subset \mathbb{R}$ is an open interval and $f_{2} \in C^{\infty}(I)$ satisfying $f_{2}^{\prime \prime}=f_{2}$. We may also assume that $U \cap N_{1}=\emptyset$. Hence, $\left(U,\left.g\right|_{U}\right)$ is isometric to

$$
\left(I \times M_{2} \times M_{1},\left(\mathrm{~d} s^{2} \oplus_{f_{2}(s)} g_{2}\right) \oplus_{f_{1}} g_{1}\right),
$$

where $f_{1} \in C^{\infty}\left(O_{2}\right), f_{2} \in C^{\infty}(I)$ and $f_{2}^{\prime \prime}-f_{2}=0$.

We show that $f_{1} \in C^{\infty}(I)$ and $f_{1}^{\prime \prime}-f_{1}=0$. Indeed, if $X \in W_{1}^{\perp}$, we have

$$
X\left(f_{1}\right)=g\left(\nabla f_{1}, X\right)=-\frac{f_{1}}{w_{1}} g\left(W_{1}, X\right)=0,
$$


which shows that $f_{1} \in C^{\infty}(I)$. Moreover, for $y \in O_{2}$ and $\left.\left.X \in W_{2}^{\perp}\right|_{y} \cap T\right|_{y} O_{2}=\left.\mathcal{D}_{2}\right|_{y}$, we have (see Lemma 4.7 and [23], Proposition 42, case (2))

$$
g\left(W_{2}, W_{2}\right) X=B\left(X, W_{2}\right) W_{2}=-R\left(X, W_{2}\right) W_{2}=\frac{H_{f_{1}}\left(W_{2}, W_{2}\right)}{f_{1}} X=\frac{f_{1}^{\prime \prime}}{f_{1}} g\left(W_{2}, W_{2}\right) X .
$$

Because rank $V_{2}^{M} \geq 2$, it follows that rank $\mathcal{D}_{2} \geq 1$ on $U$. Therefore, one may take above $X \neq 0$, which implies that $f_{1}^{\prime \prime}=f_{1}$ and proves the claim.

We also know that

$$
w_{\alpha}^{-1} W_{\alpha}=-\frac{f_{\alpha}^{\prime}}{f_{\alpha}} \partial_{s}, \quad \alpha=1,2,
$$

while

$$
0=w_{1}^{-1} w_{2}^{-1} h\left(\left(W_{1}, w_{1}\right),\left(W_{2}, w_{2}\right)\right)=h\left(\left(w_{1}^{-1} W_{1}, 1\right),\left(w_{2}^{-1} W_{2}, 1\right)\right)=\frac{f_{1}^{\prime} f_{2}^{\prime}}{f_{1} f_{2}}-1 .
$$

Writing $f_{\alpha}(s)=A_{\alpha} \cosh (s)+B_{\alpha} \sinh (s)$, the above means that $A_{1} A_{2}-B_{1} B_{2}=0$. Since $\left(A_{i}, B_{i}\right) \neq(0,0), i=1,2$, we may rescale, if necessary, the metrics of $\left(M_{1}, g_{1}\right)$ and $\left(M_{2}, g_{2}\right)$ so as to guarantee that either a) $A_{1}^{2}-B_{1}^{2}=+1$ and $A_{2}^{2}-B_{2}^{2}=-1$ or b) $A_{1}^{2}-B_{1}^{2}=-1$ and $A_{2}^{2}-B_{2}^{2}=+1$. But since $\left.h\right|_{V_{2}}$ is positive definite, $\left\|\left(W_{2}, w_{2}\right)\right\|_{h}^{2} \geq 0$ and hence

$$
0 \leq\left\|\left(w_{2}^{-1} W_{2}, 1\right)\right\|_{h}^{2}=\left(\frac{f_{2}^{\prime}(s)}{f_{2}(s)}\right)^{2}-1,
$$

which implies that $\left|A_{2}\right| \leq\left|B_{2}\right|$, so only Case a) is possible.

It then follows easily that on $I$,

$$
f_{1}^{2}-f_{2}^{2}=1
$$

Thus there is $s_{0} \in \mathbb{R}$ such that if one writes $\tilde{I}=I-s_{0}$, then for all $\tilde{s} \in \tilde{I}$,

$$
\begin{aligned}
& f_{1}\left(\tilde{s}+s_{0}\right)=\cosh (\tilde{s})=: \tilde{f}_{1}(\tilde{s}), \\
& f_{2}\left(\tilde{s}+s_{0}\right)=\sinh (\tilde{s})=: \tilde{f}_{2}(\tilde{s}) .
\end{aligned}
$$

Since $\left(I \times M_{2} \times M_{1}, \mathrm{~d} s^{2} \oplus_{f_{2}(s)} g_{2} \oplus_{f_{1}(s)} g_{1}\right)$ is isometric to $\left(\tilde{I} \times M_{2} \times M_{1}, \mathrm{~d} s^{2} \oplus_{\tilde{f}_{2}(s)}\right.$ $g_{2} \oplus_{\tilde{f}_{1}(s)} g_{1}$ ), we have proved (LW2) of Proposition 4.3 when rank $V_{2}^{M} \geq 2$.

Next we consider the case where $x \in N_{1}$. In this situation, Lemma 4.13 case (ii) implies (after maybe shrinking $U$ around $x$ ) that $\left(\mathrm{O}_{2},\left.g\right|_{\mathrm{O}_{2}}\right)$ is isometric to an open subset of $\left(\mathbb{H}^{k}, \mathbf{g}_{-1}^{k}\right)$. Moreover, by the proof of Lemma 4.13 case (ii), we may assume that $\left(O_{2} \backslash\{x\},\left.g\right|_{O_{2} \backslash\{x\}}\right)$ is isometric to (] $0, \epsilon\left[\times S^{k-1},\left.\mathrm{~d} s^{2} \oplus_{\sinh (s)} g\right|_{S^{k-1}}\right)$ through the map $F$ introduced there.

We show that $\tilde{f}_{1}:=f_{1} \circ F \in C^{\infty}(] 0, \epsilon[)$ and $\tilde{f}_{1}^{\prime \prime}-\tilde{f}_{1}=0$. Indeed, if $X \in W_{1}^{\perp}$, then $X\left(f_{1}\right)=0$ by (8). Hence, in particular, if $\left.\tilde{X} \in T\right|_{F^{-1}(y)} S^{k-1}$ for $y \in U \backslash\{x\}$, then $\tilde{X} \perp \partial_{s}$ i.e. $F_{*}(\tilde{X}) \perp W_{1}$, and hence $\tilde{X}\left(\tilde{f}_{1}\right)=0$. This shows that $\tilde{f}_{1}$ is constant on each set $\left.\{s\} \times S^{k-1}, s \in\right] 0, \epsilon\left[\right.$, since $S^{k-1}$ is connected and thus $\tilde{f}_{1} \in C^{\infty}(] 0, \epsilon[)$. From Eq. (9) one then infers that $\tilde{f}_{1}^{\prime \prime}-\tilde{f}_{1}=0$.

Thus for some $A_{1}, B_{1} \in \mathbb{R}, \tilde{f}_{1}(s)=A_{1} \cosh (s)+B_{1} \sinh (s)$. Recall that $-\frac{\nabla f_{1}}{f_{1}} \partial_{s}=$ $w_{1}^{-1} W_{1}$ where

$$
(0,1)=\left(W_{1}, w_{1}\right)+\left(W_{2}, w_{2}\right)
$$


with $\left(W_{1}, w_{1}\right) \in V_{1},\left(W_{2}, w_{2}\right) \in V_{2}$. Since $\left\|\left(W_{2}, w_{2}\right)\right\|_{h}^{2} \geq 0,\|(0,1)\|_{h}^{2}=-1$ and $\nabla f_{1}=\tilde{f}_{1}^{\prime} F_{*}\left(\partial_{s}\right)$, it follows that

$$
\left(\frac{\tilde{f}_{1}^{\prime}}{\tilde{f}_{1}}\right)^{2}-1=\left\|\left(w_{1}^{-1} W_{1}, 1\right)\right\|_{h}^{2}<0
$$

and hence $\left|B_{1}\right|<\left|A_{1}\right|$. Then, one can normalize $A_{1}, B_{1}$ such that $A_{1}^{2}-B_{1}^{2}=-1$ and by eventually replacing, as before, $s$ by $s+s_{0}$, for some $s_{0} \in \mathbb{R}$ (these operations just rescale the metric $g_{1}$ by a constant), one gets $\tilde{f}_{1}(s)=\cosh (s)$.

If $d(y):=d(x, y)$ is the distance function of $\left(O_{2},\left.g\right|_{O_{2}}\right)$ from $x$, then clearly $s=$ $d(F(s, u))$ for $(s, u) \in] 0, \epsilon\left[\times S^{k-1}\right.$, which implies that $f_{1}(y)=\cosh (d(y))$ for all $y \in O_{2}$. Thus we have arrived at (LW3) when $\operatorname{rank} V_{2}^{M} \geq 2$.

It remains to provide an argument for the case rank $V_{2}^{M}=1$. Let $x \in M$. By Lemma 4.13 case (i) with $\alpha=1$, one gets that $\left(O_{1},\left.g\right|_{O_{1}}\right)$ is isomorphic to a warped product $\left(I \times M_{1}, \mathrm{~d} s^{2} \oplus_{f_{1}(s)} g_{1}\right)$ where $f_{1} \in C^{\infty}(I)$ satisfies $f_{1}^{\prime \prime}-f_{1}=0$. Then exactly the same argument as above, replacing $\tilde{f}_{1}$ by $f_{1}$, leads to $(10)$ and to the conclusion that we may take $f_{1}(s)=\cosh (s)$ (after scaling the metric $g_{1}$ by a constant). Hence we have (LW2) and the proof of Proposition 4.3 complete in this case.

Proof of Theorem 4.1. Let us now assume that $(M, g)$ is complete and simply connected and use the notation of the above proof of Proposition 4.3.

By Theorem 3.4 we have in the proof of Proposition 4.3 that $\left(O_{2},\left.g\right|_{O_{2}}\right)$ and $\left(M_{1}, g_{1}\right)$ are complete and simply connected. Since $N_{1} \neq \emptyset$, we may further assume that $x \in N_{1} \cap O_{2}$.

Then if $k:=\operatorname{rank} V_{2}^{M} \geq 2$, the argument leading to (LW3) shows, since one may take $\epsilon=+\infty$ there, that $\left(O_{2},\left.g\right|_{O_{2}}\right)$ is isometric to $\left(\mathbb{H}^{k}, \mathbf{g}_{-1}^{k}\right)$ and that $f_{1}$ can be chosen to be $\cosh (d(\cdot))$ where $d$ is the distance function on $\left(\mathbb{H}^{k}, \mathbf{g}_{-1}^{k}\right)$ from the point corresponding to $x$. This proves (WP2).

If $k=\operatorname{rank} V_{2}^{M}=1$, then in the above argument leading to (LW2), one may take $I=\mathbb{R}$ and hence we have (WP2) with $k=1$. This completes the proof of Theorem 4.1 .

4.2.2. Case $V_{1} \cap V_{2} \neq\{0\}$. In this case, $\operatorname{dim}\left(V_{1} \cap V_{2}\right)=1$ and $V_{1} \cap V_{2}$ is lightlike and invariant by $\mathcal{H}^{-1}$ since $V_{1}$ and $V_{2}$ are. Therefore, at every point $x \in M$ there is $\left.\left.L\right|_{x} \in T\right|_{x} M$ such that $\left.\left.V_{1}\right|_{x} \cap V_{2}\right|_{x}=\mathbb{R}\left(\left.L\right|_{x}, 1\right)$. In this way, we may choose $\left.L\right|_{x}$ locally such that $L:=\left(\left.x \mapsto L\right|_{x}\right)$ becomes a smooth locally defined vector field on $M$ and if $M$ is simply connected, $L$ can be chosen to be globally defined.

Since $(L, 1)$ is lightlike vector in $\left.T\right|_{x} M \oplus \mathbb{R}$, we have $0=\|(L, 1)\|_{h}^{2}=\|L\|_{g}^{2}-1$ i.e. $L$ is a unit vector field.

Lemma 4.14 For all $X \in T M$,

$$
\nabla_{X} L=-X+g(X, L) L .
$$

Proof. Since $V_{1} \cap V_{2}=\mathbb{R}(L, 1)$ and because $V_{1} \cap V_{2}$ is invariant under $\mathcal{H}^{-1}$, we get that $V_{1} \cap V_{2}$ is invariant under parallel transport w.r.t. $\nabla^{-1}$. This is equivalent to the fact that for any $X \in T M$ there is $\alpha(X) \in \mathbb{R}$ such that

$$
\nabla_{X}^{-1}(L, 1)=\alpha(X)(L, 1)
$$

i.e.,

$$
\left(\nabla_{X} L+X, g(X, L)\right)=\alpha(X)(L, 1)
$$


from which one gets $\alpha(X)=g(X, L)$ and thus

$$
\nabla_{X} L+X=g(X, L) L
$$

Lemma 4.15 The vector field $L$ is geodesic, the distribution $L^{\perp}$ is integrable and its integral manifolds are spherical.

Proof. By Lemma 4.14 and the fact that $\|L\|_{g}=1$, we get $\nabla_{L} L=-L+g(L, L) L=$ $-L+L=0$ so $L$ is a geodesic vector field.

Let us prove the integrability of $L^{\perp}$. If $X, Y \in L^{\perp}$, then

$$
\begin{aligned}
g([X, Y], L) & =g\left(\nabla_{X} Y-\nabla_{Y} X, L\right)=-g\left(Y, \nabla_{X} L\right)+g\left(X, \nabla_{Y} L\right) \\
& =-g(Y,-X+g(X, L) L)+g(X,-Y+g(Y, L) L) \\
& =g(Y, X)-g(X, Y)=0,
\end{aligned}
$$

i.e., $[X, Y] \in L^{\perp}$. This proves that $L^{\perp}$ is involutive and hence integrable.

Let $O$ be an integral manifold of $L^{\perp}$. If $X, Y \in \mathrm{VF}(O)$, we get

$$
g\left(\nabla_{X} Y, L\right)=-g\left(Y, \nabla_{X} L\right)=-g(Y,-X+g(X, L) L)=g(X, Y),
$$

so the second fundamental form $\mathrm{II}_{O}$ of $O$ is given by

$$
\mathrm{II}_{O}(X, Y)=g(X, Y) L, \quad X,\left.Y \in T\right|_{x} M, x \in O,
$$

which means that $O$ is umbilical.

To show that $O$ is spherical, we need to show that $\nabla_{X} L \in L^{\perp}$ for all $X \in T O$. But this is clear since $0=X g(L, L)=2 g\left(\nabla_{X} L, L\right)$. This completes the proof.

We now finish the proof of Proposition 4.3 in this case. By the previous lemma and Theorem 3.4, it follows that locally $(M, g)$ is isometric to a warped product $\left(I \times M_{1}, \mathrm{~d} s^{2} \oplus_{f} g_{1}\right)$ for some interval $I \subset \mathbb{R}$ and $f \in C^{\infty}(I)$. If $(M, g)$ is complete and simply connected, then $I=\mathbb{R}$. Moreover, one has

$$
\frac{f^{\prime}}{f} X=\nabla_{X} L=-X+g(X, L) L=-X,
$$

for any $X \in L^{\perp}$. It follows that $f(s)=C e^{-s}$ for some $C \neq 0$. By rescaling the metric $g_{1}$ by a constant, we may assume that $C=1$. This leads us to the case (LW1).

\subsection{Proof of Proposition 4.4.}

4.3.1. Case $V_{1} \cap V_{2} \neq\{0\}$ : Suppose $(M, g)=\left(I \times M_{1}, \mathrm{~d} s^{2} \oplus_{e^{-s}} g_{1}\right)$. Let $L:=\partial_{s}$, $f(s)=e^{-s}$ and compute that for all $Y \in L^{\perp}$,

$$
\nabla_{Y} L=\frac{f^{\prime}}{f} Y=-Y
$$

and $\nabla_{L} L=0$, so for every $X \in T M$

$$
\nabla_{X} L=-X+g(X, L) L .
$$

Define a one-dimensional subbundle of $\pi_{T M \oplus \mathbb{R}}$ whose fibers are $V_{1}:=\mathbb{R}(L, 1)$. Then $V_{1}$ is light-like and for every $X \in T M$,

$$
\nabla_{X}^{-1}(L, 1)=\left(\nabla_{X} L+X, g(X, L)\right)=g(X, L)(L, 1),
$$

which shows that $V_{1}$ is invariant by parallel transport with respect to $\nabla^{-1}$. In particular, $V_{1}$ is invariant with respect to $\mathcal{H}^{-1}$ and therefore $\mathcal{H}^{-1}$ is reducible. This proves that $\mathcal{H}^{-1}$ is reducible if $(M, g)$ is of the form (LW1). 
4.3.2. Case $V_{1} \cap V_{2}=\{0\}$ : Assume first that $(M, g)=\left(I \times M_{2} \times M_{1}\right.$, $\mathrm{d} s^{2} \oplus_{\sinh (s)}$ $\left.g_{2} \oplus_{\cosh (s)} g_{1}\right)$. Here $I \subset \mathbb{R}$ is an interval not containing 0 . Define for every $x=$ $\left(s, x_{2}, x_{1}\right) \in M$,

$$
\left.V_{1}\right|_{x}:=\left.\mathbb{R}\left(\left.W_{1}\right|_{x}, w_{1}(x)\right) \oplus\left(\left.T\right|_{x_{1}} M_{1} \times\{0\}\right) \subset T\right|_{x} M \oplus \mathbb{R},
$$

where

$$
\left(\left.W_{1}\right|_{x}, w_{1}(x)\right):=\cosh (s)\left(-\sinh (s) \partial_{s}, \cosh (s)\right) .
$$

We prove that $V_{1}$ is invariant under $\mathcal{H}^{-1}$.

Indeed, let $\left.X \in T\right|_{x_{1}} M_{1}, Y \in \operatorname{VF}\left(M_{1}\right)$ and $\left.Z \in T\right|_{\left(s, x_{2}\right)}\left(I \times M_{2}\right)$. Then

$$
\begin{aligned}
\nabla_{X}^{-1}(Y, 0) & =\left(\nabla_{X} Y, g(X, Y)\right)=\left(\nabla_{X}^{g_{1}} Y-g(X, Y) \tanh (s) \partial_{s}, g(X, Y)\right) \\
& =\left(\nabla_{X}^{g_{1}} Y, 0\right)+\left.\frac{g(X, Y)}{\cosh ^{2}(s)}\left(\left.W_{1}\right|_{x}, w_{1}(x)\right) \in V_{1}\right|_{x}, \\
\nabla_{Z}^{-1}(Y, 0) & =\left(\nabla_{Z} Y, g(Z, Y)\right)=\left.\left(g\left(Z, \partial_{s}\right) \tanh (s) Y, 0\right) \in V_{1}\right|_{x},
\end{aligned}
$$

and if $\left.U \in T\right|_{x_{2}} M_{2}$,

$$
\begin{aligned}
\nabla_{X}^{-1}\left(W_{1}, w_{1}\right) & =\left(-\sinh ^{2}(s) X+w_{1} X, 0\right)=\left.(X, 0) \in V_{1}\right|_{x}, \\
\nabla_{U}^{-1}\left(W_{1}, w_{1}\right) & =\left(-\cosh ^{2}(s) U+w_{1} U, 0\right)=\left.(0,0) \in V_{1}\right|_{x}, \\
\nabla_{\partial_{s}}^{-1}\left(W_{1}, w_{1}\right) & =\left(\left(-\sinh ^{2}(s)-\cosh ^{2}(s)\right) \partial_{s}+w_{1} \partial_{s}, 2 \cosh (s) \sinh (s)\right. \\
& -\sinh (s) \cosh (s))=\sinh (s)\left(-\sinh (s) \partial_{s}, \cosh (s)\right)=\tanh (s)\left(W_{1}, w_{1}\right),
\end{aligned}
$$

which impies that $\left.\nabla_{X}^{-1}\left(W_{1}, w_{1}\right) \in V_{1}\right|_{x}$. These formulas show that for all $X \in T M$ and $Y \in \Gamma\left(\pi_{V_{1}}\right)$, one has $\nabla_{X}^{-1} Y \in \Gamma\left(\pi_{V_{1}}\right)$. Thus $V_{1}$ is invariant under parallel transport w.r.t. $\nabla^{-1}$ and therefore it is invariant under $\mathcal{H}^{-1}$. This proves that $\mathcal{H}^{-1}$ is reducible if $(M, g)$ is of the form (LW2).

Consider then the case where $(M, g)=\left(O \times M_{1}, \mathbf{g}_{-1}^{k} \oplus_{\cosh (d(\cdot))} \oplus g_{1}\right)$, where $O$ is a normal neighbourhood of a point $x_{0} \in \mathbb{H}^{k}$ and $d(x)=d\left(x, x_{0}\right)$ is the distance function from $x_{0}$ in $\mathbb{H}^{k}$.

Observe that $\left(O \backslash\left\{x_{0}\right\}, \mathbf{g}_{-1}^{k}\right)$ is isometric to (] $0, \epsilon\left[\times S^{k-1}, \mathrm{~d} s^{2} \oplus_{\sinh (s)} \mathbf{g}_{1}^{k-1}\right)$, for $\epsilon>0$ or $\epsilon=+\infty$ and $d(x)=s$ if $x \neq x_{0}$ corresponds to $\left.(s, y) \in\right] 0, \epsilon\left[\times S^{k-1}\right.$. Choosing above $\left(M_{2}, g_{2}\right)=\left(S^{k-1}, \mathbf{g}_{1}^{k-1}\right)$, we conclude that $\left.\mathcal{H}^{-1}\right|_{] 0, \epsilon\left[\times M_{2} \times M_{1}\right.}$ is reducible and hence by continuity, $\mathcal{H}^{-1}$ is reducible on $(M, g)$. We conclude that if $(M, g)$ is of the form (LW3), then $\mathcal{H}^{-1}$ is reducible and hence completes the proof of Proposition 4.4 .

The proof of sufficiency of Theorem 4.1 now follows immediately from the above, since one could take $O=\mathbb{H}^{k}$, the exponential map of $\mathbb{H}^{k}$ at $x_{0}$ being a diffeomorphism of $\left.T\right|_{x_{0}} \mathbb{H}^{k}$ onto $\mathbb{H}^{k}$.

\section{REFERENCES}

[1] Alouges, F., Chitour Y. and Long, R. A motion planning algorithm for the rolling-body problem, IEEE Trans. on Robotics 26 (2010), no. 5, 827-836.

[2] Agrachev A. and Sachkov Y., An Intrinsic Approach to the Control of Rolling Bodies, Proceedings of the Conference on Decision and Control, Phoenix, 1999, pp. 431 - 435, vol.1.

[3] Agrachev, A. and Sachkov, Y., Control Theory from the Geometric Viewpoint, Encyclopaedia of Mathematical Sciences, 87. Control Theory and Optimization, II. Springer-Verlag, Berlin, 2004.

[4] Alekseevsky, D. V., Cortés, V., Galaev, A. S. and Leistner, T. Cones over pseudo-Riemannian manifolds and their holonomy. J. Reine Angew. Math. 635 (2009), 23-69.

[5] Berger, M., Sur les groupes d'holonomie homogène des variétés à connexion affine et des variétés riemanniennes, Bulletin de la Société Mathématique de France 83 (1955), 279-330. 
[6] Bryant, R., Geometry of Manifolds with Special Holonomy: "100 Years of Holonomy", Contemporary Mathematics, Volume 395, 2006.

[7] Bryant, R. and Hsu, L., Rigidity of integral curves of rank 2 distributions, Invent. Math. 114 (1993), no. 2, 435-461.

[8] Chaplygin, S. A., On some feasible generalization of the theorem of area, with an application to the problem of rolling spheres (in Russian), Mat. Sbornik XX, 1-32. English translation in Regul. Chaotic Dyn. 17 (2012), no. 2, 199-217.

[9] Chaplygin, S. A., On the rolling of a sphere on a horizontal plane (in Russian), Mat. Sbornik XXIV, 139-168. English translation in Regul. Chaotic Dyn. 7 (2002), no. 2, 131-148.

[10] Chelouah, A. and Chitour, Y., On the controllability and trajectories generation of rolling surfaces, Forum Math. 15 (2003) 727-758.

[11] Chitour, Y. and Kokkonen, P., Rolling Manifolds: Intrinsic Formulation and Controllability, arXiv:1011.2925v2, 2011.

[12] Chitour, Y. and Kokkonen, P., Rolling Manifolds on Space Forms. Ann. Inst. H. Poincaré Anal. Non Linéaire 29 (2012), no. 6, 927-954.

[13] Di Scala, A.J. and Olmos, C., The geometry of homogeneous submanifolds of hyperbolic space. Math. Z. 237(1) (2001) 199-209.

[14] Godoy Molina, M., Grong, E., Markina, I. and Silva Leite, F., An intrinsic formulation of the problem on rolling manifolds. J. Dyn. Control Syst. 18 (2012), no. 2, 181-214.

[15] Godoy Molina, M. and Grong, E., Geometric conditions for the existence of an intrinsic rolling. To appear Commun. Pure Appl. Anal.

[16] Grong, E., Controllability of rolling without twisting or slipping in higher dimensions, SIAM J. Control Optim. 50-4 (2012), pp. 2462-2485.

[17] Hiepko, S. Eine innere Kennzeichnung der verzerrten Produkte, Math. Ann. 241 (1979), no. $3,209-215$.

[18] Jurdjevic, V. and Zimmerman, J., Rolling sphere problems on spaces of constant curvature. Math. Proc. Cambridge Philos. Soc. 144 (2008), no. 3, 729-747.

[19] Kobayashi, S. and Nomizu, K., Foundations of Differential Geometry, Vol. I, WileyInterscience, 1996.

[20] Marigo, A. and Bicchi A., Rolling bodies with regular surface: controllability theory and applications, IEEE Trans. Automat. Control 45 (2000), no. 9, 1586-1599.

[21] Murray, R., Li, Z. and Sastry, S. A mathematical introduction to robotic manipulation, CRC Press, Boca Raton, FL, 1994.

[22] Nomizu, K., Kinematics and differential geometry of submanifolds. Tôhoku Mathematical Journal, 30 (1978), no. 4, 623-637.

[23] O'Neill, B., Semi-Riemannian Geometry with Applications to Relativity, Academic Press, 1983

[24] Sakai, T., Riemannian Geometry, Translations of Mathematical Monographs, 149. American Mathematical Society, Providence, RI, 1996.

[25] Sharpe, R.W., Differential Geometry: Cartan's Generalization of Klein's Erlangen Program, Graduate Texts in Mathematics, 166. Springer-Verlag, New York, 1997.

[26] Wu, H., On the de Rham decomposition theorem, Illinois J. Math. 8, (1964), 291-311

L2S, Université Paris-Sud XI, CNRS and Supélec, Gif-Sur-Yvette, 91192, France. E-mail address: yacine.chitour@lss.supelec.fr

L2S, Université Paris-Sud XI, CNRS and Supélec, Gif-Sur-Yvette, 91192, France. E-mail address: mauricio.godoy@gmail.com

L2S, Université Paris-Sud XI, CNRS and Supélec, Gif-sur-Yvette, 91192, France and University of Eastern Finland, Department of Applied Physics, 70211, Kuopio, FINLAND.

E-mail address: petri.kokkonen@lss.supelec.fr 